\title{
EXTINCTION RATE OF CONTINUOUS STATE BRANCHING PROCESSES IN CRITICAL LÉVY ENVIRONMENTS
}

\author{
Vincent Bansaye ${ }^{1}$, Juan Carlos Pardo ${ }^{2, *}$ and Charline Smadi $^{3,4}{ }^{(0)}$
}

\begin{abstract}
We study the speed of extinction of continuous state branching processes in a Lévy environment, where the associated Lévy process oscillates. Assuming that the Lévy process satisfies Spitzer's condition, we extend recent results where the associated branching mechanism is stable. The study relies on the path analysis of the branching process together with its Lévy environment, when the latter is conditioned to have a non-negative running infimum. For that purpose, we combine the approach developed in Afanasyev et al. [2], for the discrete setting and i.i.d. environments, with fluctuation theory of Lévy processes and a result on exponential functionals of Lévy processes due to Patie and Savov $[28]$.
\end{abstract}

Mathematics Subject Classification. 60J80, 60G51, 60H10, 60K37.

Received March 24, 2020. Accepted June 29, 2021.

\section{INTRODUCTION AND MAIN RESULTS}

In this manuscript, we are interested in continuous state branching processes (CSBPs) which can be considered as the continuous analogues of Galton-Watson (GW) processes in time and state space. Formally speaking, a process in this class is a strong Markov process taking values in $[0, \infty]$, where 0 and $\infty$ are absorbing states, and satisfying the branching property, that is to say the law of the process started from $x+y$ is the same as the law of the sum of two independent copies of the same process issued respectively from $x$ and $y$. CSBPs have been introduced by Jirina [20] in the late fifties, of the last century, and since then they have been deeply studied by many authors including Bingham [9], Grey [17], Grimvall [18], Lamperti [22, 23], to name but a few. An interesting feature of CSBPs is that they can be obtained as scaling limits of GW processes, see for instance Grimvall [18] and Lamperti [23].

Galton-Watson processes in random environment (GWREs) were introduced by Smith and Wilkinson [29] in the late sixties of the last century. This type of processes has attracted considerable interest in the last decade, see for instance $[1-3,12]$ and the references therein. Indeed, such a family of processes provides a richer class of population models, taking into account the effect of the environment on demographic parameters and

Keywords and phrases: Continuous state branching processes, Lévy processes conditioned to stay positive, random environment, Spitzer's condition, extinction, long time behaviour.

1 CMAP, École Polytechnique, Route de Saclay, 91128 Palaiseau Cedex, France.

2 CIMAT A.C. Calle Jalisco s/n. C.P. 36240, Guanajuato, Mexico.

3 Université Clermont Auvergne, Irstea, UR LISC, Centre de Clermont-Ferrand, 9 avenue Blaise Pascal CS 20085, 63178 Aubière, France.

4 Complex Systems Institute of Paris Ile-de-France, 113 rue Nationale, Paris, France.

* Corresponding author: jcpardo@cimat.mx

(C) The authors. Published by EDP Sciences, SMAI 2021 
letting new phenomena appear. In particular, the classification of the asymptotic behaviour of rare events, such as survival probability and large deviations, is much more complex, since it may combine environmental and demographical stochasticities.

Scaling limits of GWREs have been studied by Kurtz [21] in the continuous path setting and, more recently, by Bansaye and Simatos [6] and Bansaye et al. [4] for a larger class of processes in random environment that includes CSBPs. The limiting processes satisfy the Markov property and the quenched branching property, i.e. conditionally on the environment the process started from $x+y$ is distributed as the sum of two independent copies of the same process issued respectively from $x$ and $y$. Such processes may be thought of, and therefore called, CSBPs in random environment. An interesting subclass of the aforementioned family of processes arises from several scalings of discrete models in i.i.d. environments (see for instance $[5,6,11]$ ) and can be characterized by a stochastic differential equation (SDE) where the linear term is driven by a Lévy process. Such a Lévy process captures the effect of the environment on the mean offspring distribution of individuals. A process in this subclass is known as CSBP in Lévy environment and its construction has been given by He et al. [19] and by Palau and Pardo [26], independently, as the unique strong solution of a SDE which will be specified below.

The study of the long term behaviour of CSBPs in Lévy environment has attracted considerable attention recently, see for instance $[5,11,24,25,27]$. In all these manuscripts, the speed of extinction has been computed for the case where the associated branching mechanism is stable since the survival probability can be expressed explicitly in terms of exponential functionals of Lévy processes. In [11], Böinghoff and Hutzenthaler have studied the Feller diffusion case in a Brownian environment and exploited the explicit density of the exponential functional of a Brownian motion with drift. Then Bansaye et al. [5] studied the long term behaviour for stable branching mechanisms where the random environment is driven by a Lévy process with bounded variation paths. Palau et al. [27] and $\mathrm{Li}$ and $\mathrm{Xu}$ [24] extended these results and obtained the extinction probability for stable CSBPs in a general Lévy environment.

Our aim is to relax the assumption that the branching mechanism is stable, that is to say, we are interested in studying the survival probability for a larger class of branching mechanisms associated to CSBPs in Lévy environments. Here we focus on the critical case, more precisely in oscillating Lévy environments satisfying the so-called Spitzer's condition which is a well-known assumption in fluctuation theory of Lévy processes (see assumption (H1) below). In order to do so, we use two main tools in our arguments: fluctuation theory and the asymptotic behaviour of exponential functionals of Lévy processes satisfying Spitzer's condition. We follow the point of view of Afanasyev et al. [2] in the discrete time setting, to deduce pathwise relationships between the dynamics of the CSBP in random environment and the Lévy process driving the random environment on the survival event. More precisely, we prove that the survival of the process is strongly related to its survival up to the time when the random environment reaches its running infimum. Then, we decompose its paths into two parts, the pre-infimum and post-infimum processes. If the process survives until the time when the random environment reaches its running infimum, then it has a positive probability to survive after this time and, consequently, it evolves in a "favorable" environment. As we will see below, the global picture stays unchanged compared to [2] but new difficulties arise in the continuous space setting. In particular, the state 0 can be polar and the process might become very close to 0 but never reach this point. To focus on the absorption event, we use Grey's condition which guarantees that 0 is accessible. Another difficulty arises at the upper bound for the probability of survival. Indeed, in the discrete setting, to bound the probability of survival we can use the fact that the probability that the process survives at times when the environment reaches a local minimum is equal to the probability that the current population size is bigger or equal than 1 at times when the environment reaches a local minimum. Then Chebyshev or Markov inequality will help to obtain a suitable upper bound. In the continuous setting, this strategy is not helpful. In fact, it is suitable to perform good estimates for the probability that the process survives at times when the environment reaches a local minimum. In order to do so explicit knowledge of the probability of extinction is required but the latter can only be derived in few cases, even in the case when the environment is fixed. When the environment is fixed, good estimates of such probability can be derived when the branching mechanism is regularly varying at $\infty$ with index $\vartheta \in(1,2)$ or possesses a Blumenthal-Getoor index bigger than one. In our case, the latter type of estimates cannot be 
obtained due to the environment as we will explain below. So in order to overcome these difficulties, we impose some assumptions on the branching mechanism and on the environment which are not so restrictive.

\subsection{CSBPs in a Lévy environment}

Let $\left(\Omega^{(b)}, \mathcal{F}^{(b)},\left(\mathcal{F}_{t}^{(b)}\right)_{t \geq 0}, \mathbb{P}^{(b)}\right)$ be a filtered probability space satisfying the usual hypothesis and introduce a $\left(\mathcal{F}_{t}^{(b)}\right)_{t \geq 0}$-adapted standard Brownian motion $B^{(b)}=\left(B_{t}^{(b)}, t \geq 0\right)$ and an independent $\left(\mathcal{F}_{t}^{(b)}\right)_{t \geq 0}$-adapted Poisson random measure $N^{(b)}(\mathrm{d} s, \mathrm{~d} z, \mathrm{~d} u)$ defined on $\mathbb{R}_{+}^{3}$, with intensity $\mathrm{d} s \mu(\mathrm{d} z) \mathrm{d} u$. The measure $\mu$ is concentrated on $(0, \infty)$ and in the whole paper we assume that

$$
\int_{(0, \infty)}\left(z \wedge z^{2}\right) \mu(\mathrm{d} z)<\infty
$$

which guarantees non-explosivity (see Lem. A.1 in the Appendix for the proof of this fact). We denote by $\widetilde{N}^{(b)}$ the compensated measure of $N^{(b)}$.

According to Dawson and Li [15], we can define $Y=\left(Y_{t}, t \geq 0\right)$, a CSBP, as the unique strong solution of the following SDE

$$
Y_{t}=Y_{0}-\psi^{\prime}(0+) \int_{0}^{t} Y_{s} \mathrm{~d} s+\int_{0}^{t} \sqrt{2 \gamma^{2} Y_{s}} \mathrm{~d} B_{s}^{(b)}+\int_{0}^{t} \int_{(0, \infty)} \int_{0}^{Y_{s-}} z \tilde{N}^{(b)}(\mathrm{d} s, \mathrm{~d} z, \mathrm{~d} u),
$$

where $\gamma \geq 0$ and $\psi^{\prime}(0+) \in \mathbb{R}$, denotes the right derivative at 0 of the so-called branching mechanism associated to $Y$ which satisfies the celebrated Lévy-Khintchine formula, i.e.

$$
\psi(\lambda)=\lambda \psi^{\prime}(0+)+\gamma^{2} \lambda^{2}+\int_{(0, \infty)}\left(e^{-\lambda x}-1+\lambda x\right) \mu(\mathrm{d} x), \quad \text { for } \quad \lambda \geq 0 .
$$

For the random environment, we consider $\left(\Omega^{(e)}, \mathcal{F}^{(e)},\left(\mathcal{F}_{t}^{(e)}\right)_{t \geq 0}, \mathbb{P}^{(e)}\right)$ a filtered probability space satisfying the usual hypothesis and a $\left(\mathcal{F}_{t}^{(e)}\right)$-Lévy process $K=\left(K_{t}, t \geq 0\right)$ which is defined as follows

$$
K_{t}=\alpha t+\sigma B_{t}^{(e)}+\int_{0}^{t} \int_{\mathbb{R} \backslash(-1,1)}\left(e^{z}-1\right) N^{(e)}(\mathrm{d} s, \mathrm{~d} z)+\int_{0}^{t} \int_{(-1,1)}\left(e^{z}-1\right) \widetilde{N}^{(e)}(\mathrm{d} s, \mathrm{~d} z)
$$

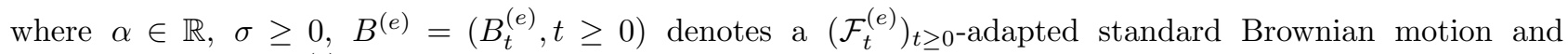
$N^{(e)}(\mathrm{d} s, \mathrm{~d} z)$ is a $\left(\mathcal{F}_{t}^{(e)}\right)_{t \geq 0}$-adapted Poisson random measure on $\mathbb{R}_{+} \times \mathbb{R}$ with intensity $\mathrm{d} s \pi(\mathrm{d} y)$, which is independent of $B^{(e)}$. The measure $\pi$ is concentrated on $\mathbb{R} \backslash\{0\}$ and fulfills the following integral condition

$$
\int_{\mathbb{R}}\left(1 \wedge z^{2}\right) \pi(\mathrm{d} z)<\infty
$$

In our model, the population size has no impact on the evolution of the environment and we are considering independent processes for demography and environment. More precisely, we work now on the product space $\left(\Omega, \mathcal{F},(\mathcal{F})_{t \geq 0}, \mathbb{P}\right)$, where $\Omega=\Omega^{(e)} \times \Omega^{(b)}, \mathcal{F}=\mathcal{F}^{(e)} \otimes \mathcal{F}^{(b)}$, and $\mathcal{F}_{t}=\mathcal{F}_{t}^{(e)} \otimes \mathcal{F}_{t}^{(b)}$ for $t \geq 0, \mathbb{P}=\mathbb{P}^{(e)} \otimes \mathbb{P}^{(b)}$ and we make the direct extension of $B^{(b)}, N^{(b)}, B^{(e)}, N^{(e)}$ and $K$ to $\Omega$ by projection respectively on $\Omega^{(b)}$ and $\Omega^{(e)}$. In particular, $\left(B^{(e)}, N^{(e)}\right)$ is independent of $\left(B^{(b)}, N^{(b)}\right)$. 
Letting $Z_{0} \in[0, \infty)$ a.s., a CSBP in a Lévy environment $Z$ can be defined as the unique non-negative strong solution of the following SDE,

$$
\begin{aligned}
Z_{t}=Z_{0}-\psi^{\prime}(0+) \int_{0}^{t} Z_{s} \mathrm{~d} & +\int_{0}^{t} \sqrt{2 \gamma^{2} Z_{s}} \mathrm{~d} B_{s}^{(b)} \\
& +\int_{0}^{t} \int_{(0, \infty)} \int_{0}^{Z_{s-}} z \tilde{N}^{(b)}(\mathrm{d} s, \mathrm{~d} z, \mathrm{~d} u)+\int_{0}^{t} Z_{s-} \mathrm{d} K_{s} .
\end{aligned}
$$

According to He et al. [19] and Palau and Pardo [26], pathwise uniqueness and strong existence hold for this SDE. Actually, Palau and Pardo also considered the case when $\psi^{\prime}(0+)=-\infty$, and obtained that the corresponding SDE has a unique strong solution up to explosion and by convention here it is identically equal to $+\infty$ after the explosion time. It turns out that (1.1) is a sufficient condition to conclude that the process $Z$ is conservative or in other words that it does not explode in finite time. The conservativeness was first observed by Palau and Pardo in [25] (see Prop. 1) in the case when the random environment is driven by a Brownian motion. A similar result also holds in our context: if (1.1) holds then

$$
\mathbb{P}_{z}\left(Z_{t}<\infty\right)=1, \quad \text { for any } \quad t \geq 0,
$$

and any $z \geq 0$ where $\mathbb{P}_{z}$ denotes the law of the process $Z$ starting from $z \geq 0$. The proof follows from similar arguments as those used in [25] and is deferred to the Appendix (see Lem. A.1).

The analysis of the process $Z$ is deeply related to the behaviour and fluctuations of the Lévy process $\bar{K}$, defined on the same filtration as $K$, which provides a quenched martingale. We set

$$
\bar{K}_{t}=\bar{\alpha} t+\sigma B_{t}^{(e)}+\int_{0}^{t} \int_{(-1,1)} z \widetilde{N}^{(e)}(\mathrm{d} s, \mathrm{~d} z)+\int_{0}^{t} \int_{\mathbb{R} \backslash(-1,1)} z N^{(e)}(\mathrm{d} s, \mathrm{~d} z),
$$

where

$$
\bar{\alpha}:=\alpha-\psi^{\prime}(0+)-\frac{\sigma^{2}}{2}-\int_{(-1,1)}\left(e^{z}-1-z\right) \pi(\mathrm{d} z)
$$

and we obtain the following statement.

Proposition 1.1. For $\mathbb{P}^{(e)}$ almost every $w^{(e)} \in \Omega^{(e)},\left(\exp \left\{-\bar{K}_{t}\left(w^{(e)},.\right)\right\} Z_{t}\left(w^{(e)},.\right): t \geq 0\right)$ is a $\left(\Omega^{(b)}, \mathcal{F}^{(b)}, \mathbb{P}^{(b)}\right)$ martingale and for any $t \geq 0$ and $z \geq 0$,

$$
\mathbb{E}_{z}\left[Z_{t} \mid K\right]=z e^{\bar{K}_{t}}, \quad \mathbb{P} \text {-a.s. }
$$

The proof is deferred to the Appendix. In other words, the process $\bar{K}$ plays an analogous role as the random walk associated to the logarithm of the offspring means in the discrete time framework and leads to the usual classification for the long time behaviour of branching processes. We say that the process $Z$ is subcritical, critical or supercritical accordingly as $\bar{K}$ drifts to $-\infty$, oscillates or drifts to $+\infty$. We refer to $[5,11,24,27]$ for asymptotic results under different regimes. We observe that in the critical case and contrary to the discrete framework, the process may oscillate between 0 and $\infty$ a.s., see for instance [5].

\subsection{Properties of the Lévy environment}

Recall that $\bar{K}=\left(\bar{K}_{t}, t \geq 0\right)$ denotes the real valued Lévy process defined in (1.4). That is to say $\bar{K}$ has stationary and independent increments with càdlàg paths. For simplicity, we denote by $\mathbb{P}_{x}^{(e)}$ (resp. $\mathbb{E}_{x}^{(e)}$ ) the 
probability (resp. expectation) associated to the process $\bar{K}$ starting from $x \in \mathbb{R}$, and when $x=0$, we use the notation $\mathbb{P}^{(e)}$ for $\mathbb{P}_{0}^{(e)}$ (resp. $\mathbb{E}^{(e)}$ for $\mathbb{E}_{0}^{(e)}$ ), i.e.

$$
\mathbb{P}_{x}^{(e)}\left(\bar{K}_{t} \in B\right)=\mathbb{P}^{(e)}\left(\bar{K}_{t}+x \in B\right), \quad \text { for } \quad B \in \mathcal{B}(\mathbb{R}) .
$$

We assume in the sequel that $\bar{K}$ is not a compound Poisson process.

In what follows, we assume a general condition which is known as Spitzer's condition in fluctuation theory of Lévy processes, i.e.

$$
\frac{1}{t} \int_{0}^{t} \mathbb{P}^{(e)}\left(\bar{K}_{s} \geq 0\right) \mathrm{d} s \longrightarrow \rho \in(0,1), \quad \text { as } \quad t \rightarrow \infty
$$

Spitzer's condition implies that $\bar{K}$ oscillates and implicitly, from Proposition 1.1, that the process $Z$ is in the critical regime. According to Bertoin and Doney [8] condition (H1) is equivalent to

$$
\mathbb{P}^{(e)}\left(\bar{K}_{t} \geq 0\right) \longrightarrow \rho \in(0,1), \quad \text { as } \quad t \rightarrow \infty .
$$

Spitzer's condition is a key condition to understand the tail distribution of first passage times (see (1.8) and (1.9) for instance). Notice that if Spitzer's condition holds and $\bar{K}$ has a finite variance, then necessarily $\rho=$ 1/2. Examples of Lévy processes satisfying Spitzer's condition are the standard Brownian motion, and stable processes where $\rho \in(0,1)$ plays the role of the positivity parameter. Furthermore, any symmetric Lévy process satisfies Spitzer's condition with $\rho=1 / 2$ and any Lévy process in the domain of attraction of a stable process with positivity parameter $\rho \in(0,1)$ as $t \rightarrow \infty$, satisfies Spitzer's condition.

Our arguments on the survival event rely on the running infimum of $\bar{K}$, here denoted by $I=\left(I_{t}, t \geq 0\right)$ where

$$
I_{t}=\inf _{0 \leq s \leq t} \bar{K}_{s}, \quad t \geq 0
$$

To be more precise, we use fluctuation theory of Lévy processes reflected at their running infimum. Let us recall that the reflected process $\bar{K}-I$ is a Markov process with respect to the filtration $\left(\mathcal{F}_{t}^{(e)}\right)_{t \geq 0}$ and whose semigroup satisfies the Feller property (see for instance Proposition VI.1 in the monograph of Bertoin [7]). We denote by $\widehat{L}$ the local time of $\bar{K}-I$ at 0 in the sense of Chapter IV in [7]. Similarly to the discrete framework [2], the asymptotic analysis and the role of the initial condition involve the renewal function $\widehat{V}$ which is defined, for all $x \geq 0$, as follows

$$
\widehat{V}(x):=\mathbb{E}^{(e)}\left[\int_{[0, \infty)} \mathbf{1}_{\left\{I_{t} \geq-x\right\}} \mathrm{d} \widehat{L}_{t}\right] .
$$

The renewal function $\widehat{V}$ is subadditive, continuous and increasing and satisfies $\widehat{V}(x) \geq 0$ for $x \geq 0$ and $\widehat{V}(x)>0$ for $x>0$. See for instance the monograph of Doney [16] or Section 2 for further details about the previous facts. Under Spitzer's condition (see Thm. VI.18 in Bertoin [7]) the asymptotic behaviour of the probability that the Lévy process $\bar{K}$ remains positive, i.e. $\mathbb{P}_{x}^{(e)}\left(I_{t}>0\right)$ for $x>0$, is regularly varying at $\infty$ with index $\rho-1$ and moreover, for any $x, y>0$, we have

$$
\lim _{t \rightarrow \infty} \frac{\mathbb{P}_{x}^{(e)}\left(I_{t}>0\right)}{\mathbb{P}_{y}^{(e)}\left(I_{t}>0\right)}=\frac{\widehat{V}(x)}{\widehat{V}(y)} .
$$


In other words, we obtain that for any $x>0$,

$$
\mathbb{P}_{x}^{(e)}\left(I_{t}>0\right) \sim \widehat{V}(x) t^{\rho-1} \ell(t), \quad \text { as } \quad t \rightarrow \infty,
$$

where $\ell$ is a slowly varying function at $\infty$, that is to say, for $c>0$,

$$
\lim _{t \rightarrow \infty} \frac{\ell(c t)}{\ell(t)}=1
$$

\subsection{Main result}

We now state our main result which is devoted to the speed of extinction of CSBPs in Lévy environment under the assumption that the environment oscillates. It is important to note that the survival of the process is associated to "favorable" environments which are characterized by the running infimum of the environment, which is not too small from our assumptions.

We need some assumptions to control the effect of the environment on the event of survival. The following moment assumption is needed to guarantee the non-extinction of the process in favorable environments (see Prop. 3.4 for further details),

$$
\int^{\infty} \theta \ln ^{2}(\theta) \mu(\mathrm{d} \theta)<\infty
$$

The above condition is similar to $x \log (x)$ moment condition on the measure $\mu$, used in Proposition 2 in [25] to determine that the probability of survival of CSBP processes in Lévy environments that drifts to $+\infty$, is positive.

As we will see below, Spitzer's condition (H1) and assumption (H2) are sufficient conditions to provide a lower bound for the survival probability. In order to get the upper bound, further assumptions on the branching mechanism and the environment are required. Let

$$
\psi_{0}(\lambda):=\psi(\lambda)-\lambda \psi^{\prime}(0+), \quad \text { for } \quad \lambda \geq 0,
$$

and assume that there exist $\beta \in(0,1]$, and $C>0$ such that

$$
\psi_{0}(\lambda) \geq C \lambda^{1+\beta}, \quad \text { for } \lambda \geq 0 .
$$

Assumption (H3) allows us to control the absorption of the process for bad environments (see Lem. 4.2) and in particular, it guarantees that $\psi_{0}(\lambda)$ satisfies the so-called Grey's condition, i.e.

$$
\int^{\infty} \frac{\mathrm{d} z}{\psi_{0}(z)}<\infty
$$

which is a necessary and sufficient condition for absorption of CSBPs, see for instance [17]. Recently, He et al. [19] have shown that this condition is also necessary and sufficient for CSBPs in a Lévy environment to get absorbed with positive probability (see Thm. 4.1 in [19]). In our case since the process $\bar{K}$ oscillates and Grey's condition (1.11) is satisfied then absorption occurs a.s. according to Corollary 4.4 in [19].

Theorem 1.2. Assume that assumptions $(\mathbf{H 1})-(\mathbf{H 3})$ hold. Then there exists a positive function $c$ such that for any $z>0$,

$$
\mathbb{P}_{z}\left(Z_{t}>0\right) \sim c(z) \mathbb{P}_{1}^{(e)}\left(I_{t}>0\right) \sim c(z) \widehat{V}(1) t^{\rho-1} \ell(t), \quad \text { as } \quad t \rightarrow \infty
$$


where $\ell$ is the slowly varying function introduced in (1.9).

We point out that we only need assmptions $(\mathbf{H 1})$ and (H2) to ensure that the probability of survival of the process $Z$ satisfies

$$
\mathbb{P}_{z}\left(Z_{t}>0\right) \geq c(z) \mathbb{P}_{1}^{(e)}\left(I_{t}>0\right) \sim \widehat{V}(1) c(z) t^{\rho-1} \ell(t), \quad \text { as } \quad t \rightarrow \infty
$$

It seems quite difficult to deduce the asymptotic behaviour of the probability of survival just under assumptions (H1) and (H2), as we explain below. Let us briefly explain why stronger assumptions such as (H3) are required for the upper bound. Recall from Proposition 2 in [26], that there exists a functional $v_{t}(s, \lambda, \bar{K})$ which is the $\mathbb{P}^{(e)}$-a.s. unique solution of the backward differential equation

$$
\frac{\partial}{\partial s} v_{t}(s, \lambda, \bar{K})=e^{\bar{K}_{s}} \psi_{0}\left(v_{t}(s, \lambda, \bar{K}) e^{-\bar{K}_{s}}\right), \quad v_{t}(t, \lambda, \bar{K})=\lambda,
$$

where $\psi_{0}$ is defined as in (1.10). For simplicity of exposition, we denote by $\mathbb{P}_{(z, x)}$ (resp. $\mathbb{E}_{(z, x)}$ its expectation) for the law of the couple $(Z, \bar{K})$ started from $(z, x)$ where $z, x>0$, under $\mathbb{P}$. Thus, the functional $v_{t}(s, \lambda, \bar{K})$ determines the law of the reweighted process $\left(Z_{t} e^{-\bar{K}_{t}}, t \geq 0\right)$ as follows,

$$
\begin{aligned}
\mathbb{E}_{(z, 1)}\left[\exp \left\{-\lambda Z_{t} e^{-\bar{K}_{t}}\right\}\right] & =\mathbb{E}_{(z, 0)}\left[\exp \left\{-\lambda e^{-1} Z_{t} e^{-\bar{K}_{t}}\right\}\right] \\
& =\mathbb{E}^{(e)}\left[\exp \left\{-z v_{t}\left(0, \lambda e^{-1}, \bar{K}\right)\right\}\right]
\end{aligned}
$$

Under Grey's condition (1.11) and the previous identity, we can deduce

$$
\mathbb{P}_{(z, 1)}\left(Z_{t}>0, I_{t} \leq-y\right)=\mathbb{E}^{(e)}\left[\left(1-e^{-z \bar{v}_{t}(0, \infty, \bar{K})}\right) \mathbf{1}_{\left\{I_{t} \leq-y-1\right\}}\right], \quad \text { for } \quad y \geq 0,
$$

where $\bar{v}_{t}(0, \infty, \bar{K})$ is $\mathbb{P}^{(e)}$-a.s. finite for all $t \geq 0$, (according to Thm. 4.1 in [19]) but perhaps equals 0 . Actually, assumption (H2) guarantees that $\bar{v}_{t}(0, \infty, \bar{K})>0, \mathbb{P}^{(e)}$-a.s., for all $t>0$; and even in "favorable" environments (see Prop. 3.4). The right-hand side of (1.14) seems difficult to estimate due to the nature of the functional $\bar{v}_{t}(0, \infty, \bar{K})$. Even under the assumption that $\psi_{0}$ is regularly varying at $\infty$, it is not so clear how to handle $\bar{v}_{t}(0, \infty, \bar{K})$ due to the dependence on the environment. In the discrete setting such a probability can be estimated in terms of the infimum of the environment (which is a random walk) since the event of survival is equal to the event of the current population being bigger or equal to one. Assumption (H3) allows us to upper bound (1.14) by the exponential functional of $\bar{K}$, and to study its asymptotic behaviour.

We end our exposition with some examples where the renewal function can be computed explicitly and the previous results can be applied.

\subsection{Examples}

a) Brownian case. In the particular case when $\bar{K}$ is a standard Brownian motion starting from $x>0$, it is known that the renewal measure is proportional to the identity, i.e. $\widehat{V}(y) \propto y$, for $y \geq 0$. Moreover, Brownian motion oscillates and satisfies Spitzer's condition (H1) with $\rho=1 / 2$. Then, assuming that conditions (H2) and (H3) are fulfilled, we obtain that the CSBP in a Brownian environment satisfies

$$
\mathbb{P}_{z}\left(Z_{t}>0\right) \sim c(z) t^{-1 / 2} \ell(t), \quad \text { as } \quad t \rightarrow \infty
$$


In this particular case, we can compute the function $\ell$, i.e.

$$
\ell(t)=\int_{1}^{\infty} e^{-\frac{1}{2 t u}} \frac{\mathrm{d} u}{\sqrt{2 \pi u^{3}}}, \quad t>0
$$

which follows from the fact that the law of the infimum of a Brownian motion is given by

$$
\mathbb{P}_{1}^{(e)}\left(I_{t}>0\right)=\int_{t}^{\infty} e^{-\frac{1}{2 w}} \frac{\mathrm{d} w}{\sqrt{2 \pi w^{3}}}, \quad t>0
$$

b) Spectrally negative case. If $\bar{K}$ is a spectrally negative Lévy process, i.e. it has no positive jumps, then the renewal measure is given by the so-called scale function $W:[0, \infty) \rightarrow[0, \infty)$, which is defined as the unique continuous increasing function satisfying

$$
\int_{0}^{\infty} e^{-\lambda x} W(x) \mathrm{d} x=\frac{1}{\phi(\lambda)} \quad \text { for } \quad \lambda \geq 0
$$

where $\phi$ denotes the Laplace exponent of $\bar{K}$ which is given by $\phi(\lambda):=\log \mathbf{E}\left[e^{\lambda \bar{K}_{1}}\right]$ and satisfies the so-called Lévy-Khintchine formula. In other words, we identify the renewal function $\widehat{V}$ with the scale function $\mathrm{W}$ (i.e. $\widehat{V} \equiv W)$.

In this case, there is an interpretation of Spitzer's condition in terms of the Laplace exponent $\phi$. More precisely, from Proposition VII.6 in Bertoin [7], the spectrally negative Lévy process $\bar{K}$ satisfies Spitzer's condition with $\rho \in(0,1)$ if and only if its Laplace exponent $\phi$ is regularly varying at 0 with index $1 / \rho$. This proposition also mentions that in this case, $\rho$ is necessarily larger than $1 / 2$. Hence assuming that the Laplace exponent $\phi$ is regularly varying at 0 with index $1 / \rho$, Theorem 1 holds under the assumption that the branching mechanism satisfies $\psi_{0}(\lambda) \geq C \lambda^{1+\beta}$, for some $\beta>0$, together with condition (H2).

In the particular case where $\bar{K}$ is a spectrally negative stable process with index $\alpha \in(1,2)$, we have $W(x)=x^{\alpha-1} / \Gamma(\alpha)$, for $x \geq 0$, where $\Gamma$ denotes the so-called Gamma function. Moreover, it satisfies Spitzer's condition with $\rho=1 / \alpha$.

c) Stable case. Assume that $\alpha \in(1,2)$ and that $\bar{K}$ is a stable Lévy process with positivity parameter $\rho \in(0,1)$. It is known that the descending ladder height is a stable subordinator with parameter $\alpha(1-\rho)$ (see for instance Lem. VIII.1 in [7] and Section 2 for a proper definition of the descending ladder height) which implies that the renewal function $\widehat{V}(x)$ is proportional to $x^{\alpha(1-\rho)}$, for $x>0$. Indeed, its Laplace transform satisfies

$$
\int_{0}^{\infty} e^{-\lambda x} \widehat{V}(\mathrm{~d} x)=\frac{1}{\lambda^{\alpha(1-\rho)}}, \quad \lambda>0
$$

Hence, Theorem 1 holds under the assumption that the branching mechanism satisfies $\psi_{0}(\lambda) \geq C \lambda^{1+\beta}$, for some $\beta>0$, together with condition (H2).

The remainder of the manuscript is organized as follows. In Section 2, some preliminaries on fluctuation theory of Lévy processes are introduced, as well as the definition of their conditioned version to stay positive. Moreover some useful properties of the latter are also studied. Section 3 is devoted to the study of CSBPs in a conditioned random environment whose properties are needed for our purposes. The proof of the main result is provided in Section 4 and, finally, in Appendix A we provide the proofs of Proposition 1.1 as well as the non-explosivity of CSBPs in a Lévy random environment. 


\section{Preliminaries}

In order to provide a precise description of the relationship between the survival probability of the process $Z$ and the behaviour of the running infimum of $\bar{K}$, the description of the Lévy process $\bar{K}$ conditioned to stay positive is needed as well as the description of the process $Z$ under this conditioned random environment.

In this section, we introduce Lévy processes conditioned to stay positive as well as some of their properties that we will use in the sequel.

\subsection{Lévy processes and fluctuation theory}

Recall that $I_{t}=\inf _{0 \leq s \leq t} \bar{K}_{s}$, for $t \geq 0$, and that the reflected process $\bar{K}-I$ is a Markov process with respect to the filtration $\left(\mathcal{F}^{(e)}\right)_{t \geq 0}$ and whose semigroup satisfies the Feller property. It is important to note that the same properties are satisfied by the reflected process at its running supremum $S-\bar{K}$, where $S_{t}=\inf _{0 \leq s \leq t} \bar{K}_{s}$, since the dual process $-\bar{K}$ is also a Lévy process satisfying that for any fixed time $t>0$, the processes

$$
\left(\bar{K}_{(t-s)}-\bar{K}_{t}, 0 \leq s \leq t\right) \quad \text { and } \quad\left(-\bar{K}_{s}, 0 \leq s \leq t\right)
$$

have the same law.

We also recall that $\widehat{L}$ denotes the local time of the reflected process $\bar{K}-I$ at 0 in the sense of Chapter IV in [7]. Similarly, we denote by $L$ for the local time at 0 of $S-\bar{K}$. If 0 is regular for $(-\infty, 0)$ or regular downwards, i.e.

$$
\mathbb{P}^{(e)}\left(\tau_{0}^{-}=0\right)=1
$$

where $\tau_{0}^{-}=\inf \left\{s>0: \bar{K}_{s} \leq 0\right\}$, then 0 is regular for the reflected process $\bar{K}-I$ and then, up to a multiplicative constant, $\widehat{L}$ is the unique additive functional of the reflected process whose set of increasing points is $\left\{t: \bar{K}_{t}=\right.$ $\left.I_{t}\right\}$. If 0 is not regular downwards then the set $\left\{t: \bar{K}_{t}=I_{t}\right\}$ is discrete and we define the local time $\widehat{L}$ as the counting process of this set.

Let us denote by $\widehat{L}^{-1}$ for the inverse local time and introduce the so-called descending ladder height process $\widehat{H}$ which is defined by

$$
\widehat{H}_{t}=-I_{\widehat{L}_{t}^{-1}}, \quad t \geq 0
$$

The pair $\left(\widehat{L}^{-1}, \widehat{H}\right)$ is a bivariate subordinator, as is $\left(L^{-1}, H\right)$ where

$$
H_{t}=S_{L_{t}^{-1}}, \quad t \geq 0
$$

Both pairs are known as descending and ascending ladder processes, respectively. The Laplace transform of the descending ladder process $\left(\widehat{L}^{-1}, \widehat{H}\right)$ is such that for $\theta, \lambda \geq 0$,

$$
\mathbb{E}^{(e)}\left[\exp \left\{-\theta \widehat{L}_{t}^{-1}-\lambda \widehat{H}_{t}\right\}\right]=\exp \{-t \widehat{\kappa}(\theta, \lambda)\}, \quad t \geq 0
$$

writing $\widehat{\kappa}(\cdot, \cdot)$ for its bivariate Laplace exponent $(\kappa(\cdot, \cdot)$ for that of the ascending ladder process) which has the form

$$
\widehat{\kappa}(\theta, \lambda)=\widehat{\delta} \theta+\widehat{\mathbf{d}} \lambda+\int_{(0, \infty)^{2}}\left(1-e^{-(\theta x+\lambda y)}\right) \widehat{\mu}(\mathrm{d} x, \mathrm{~d} y),
$$


with $\widehat{\delta}, \widehat{\mathbf{d}} \geq 0$ and

$$
\int_{(0, \infty)^{2}}(x \wedge 1)(y \wedge 1) \widehat{\mu}(\mathrm{d} x, \mathrm{~d} y)<\infty
$$

Notice that both $\left(\widehat{L}^{-1}, \widehat{H}\right)$ and $\left(L^{-1}, H\right)$ have no killing terms, since we are assuming that the process $\bar{K}$ oscillates. Implicity, the Laplace exponent of $\widehat{H}$ satisfies

$$
\widehat{\kappa}(0, \lambda)=\widehat{\mathbf{d}} \lambda+\int_{(0, \infty)}\left(1-e^{-\lambda y}\right) \widehat{\eta}(\mathrm{d} y),
$$

where $\widehat{\eta}(B)=\widehat{\mu}((0, \infty), B)$ for $B \in \mathcal{B}((0, \infty))$.

An interesting connection between the distribution of the ladder processes and that of $\bar{K}$ is given by the Wiener-Hopf factorisation

$$
\mathbb{E}^{(e)}\left[e^{i \theta \bar{K}_{\mathbf{e}_{q}}}\right]=\mathbb{E}^{(e)}\left[e^{i \theta S_{\mathbf{e}_{q}}}\right] \mathbb{E}^{(e)}\left[e^{i \theta I_{\mathbf{e}_{q}}}\right],
$$

where $\mathbf{e}_{q}$ denotes an exponential random variable with parameter $q \geq 0$ which is independent of $\bar{K}$,

$$
\mathbb{E}^{(e)}\left[e^{i \theta S_{\mathbf{e}_{q}}}\right]=\frac{\kappa(q, 0)}{\kappa(q,-i \theta)} \quad \text { and } \quad \mathbb{E}^{(e)}\left[e^{i \theta I_{\mathbf{e}_{q}}}\right]=\frac{\widehat{\kappa}(q, 0)}{\widehat{\kappa}(q, i \theta)}
$$

We refer to Chapter VI in Bertoin [7] or Chapter 4 in of Doney [16] for further details on the descending ladder processes $(\widehat{H}, \widehat{L})$ as well as for the Wiener-Hopf factorisation.

Next, we consider the renewal function $\widehat{V}$ which was defined in (1.7). It is known that $\widehat{V}$ is a finite, continuous, increasing and subadditive function on $[0, \infty)$ satisfying

$$
\widehat{V}(x) \leq C_{1} x, \quad \text { for any } \quad x \geq 0,
$$

where $C_{1}$ is a finite constant (see for instance Lem. 6.4 and Sect. 8.2 in the monograph of Doney [16]). Moreover $\widehat{V}(0)=0$ if 0 is regular downwards and $\widehat{V}(0)=1$ otherwise. By a simple change of variables we can relate the definition of the renewal function $\widehat{V}$ and the descending ladder height $\widehat{H}$. Indeed, the measure induced by $\widehat{V}$ can be rewritten as follows,

$$
\widehat{V}(\mathrm{~d} x)=\mathbb{E}^{(e)}\left[\int_{0}^{\infty} \mathbf{1}_{\left\{\widehat{H}_{t} \in \mathrm{d} x\right\}} \mathrm{d} t\right] .
$$

Roughly speaking, the renewal function $\widehat{V}(x)$ "measures" the amount of time that the descending ladder height process spends on the interval $[0, x]$ and in particular induces a measure on $[0, \infty)$ which is known as the renewal measure. The latter implies

$$
\int_{[0, \infty)} e^{-\lambda x} \widehat{V}(\mathrm{~d} x)=\int_{0}^{\infty} \mathbb{E}^{(e)}\left[e^{-\lambda \widehat{H}_{t}}\right] \mathrm{d} t=\frac{1}{\widehat{\kappa}(0, \lambda)}, \quad \text { for } \quad \lambda \geq 0 .
$$

Similarly, we introduce the renewal funtion $V$ associated with the ascending ladder height induced by

$$
V(\mathrm{~d} x)=\mathbb{E}^{(e)}\left[\int_{0}^{\infty} \mathbf{1}_{\left\{H_{t} \in \mathrm{d} x\right\}} \mathrm{d} t\right]
$$


which is also a finite, continuous, increasing and subadditive function on $[0, \infty)$ such that $V(0)=0$ if 0 is regular upwards and $V(0)=1$ otherwise.

\subsection{Lévy processes conditioned to stay positive}

Let us define the probability $\mathbb{Q}_{x}$ associated to the Lévy process $\bar{K}$ started at $x>0$ and killed at time $\zeta$ when it first enters $(-\infty, 0)$, that is to say

$$
\mathbb{Q}_{x}\left[f\left(\bar{K}_{t}\right) \mathbf{1}_{\{\zeta>t\}}\right]:=\mathbb{E}_{x}^{(e)}\left[f\left(\bar{K}_{t}\right) \mathbf{1}_{\left\{I_{t}>0\right\}}\right],
$$

where $f: \mathbb{R}_{+} \rightarrow \mathbb{R}$ is measurable.

According to Lemma 1 in Chaumont and Doney [14], under the assumption that $\bar{K}$ does not drift towards $-\infty$, we have that the renewal function $\widehat{V}$ is invariant for the killed process. In other words, for all $x>0$ and $t \geq 0$

$$
\mathbb{Q}_{x}\left[\widehat{V}\left(\bar{K}_{t}\right) \mathbf{1}_{\{\zeta>t\}}\right]=\mathbb{E}_{x}^{(e)}\left[\widehat{V}\left(\bar{K}_{t}\right) \mathbf{1}_{\left\{I_{t}>0\right\}}\right]=\widehat{V}(x) .
$$

We now recall the definition of Lévy processes conditioned to stay positive as a Doob- $h$ transform. Before doing so, let us recall that $\bar{K}$ is adapted to the filtration $\left(\mathcal{F}_{t}^{(e)}\right)_{t \geq 0}$. Under the assumption that $\bar{K}$ does not drift towards $-\infty$, the law of the process $\bar{K}$ conditioned to stay positive is defined as follows, for $\Lambda \in \mathcal{F}_{t}^{(e)}$ and $x>0$,

$$
\mathbb{P}_{x}^{(e), \uparrow}(\Lambda):=\frac{1}{\widehat{V}(x)} \mathbb{E}_{x}^{(e)}\left[\widehat{V}\left(\bar{K}_{t}\right) \mathbf{1}_{\left\{I_{t}>0\right\}} \mathbf{1}_{\Lambda}\right]
$$

The term conditioned to stay positive in definition (2.9) is justified from the following convergence result due to Chaumont [13] (see also Rem. 1 in the aforementioned paper as well as Chaumont and Doney [14]) that we recall here in the particular case when the process $\bar{K}$ fulfills Spitzer's condition (H1).

Lemma 2.1. Assume that Spitzer's condition (H1) is fulfilled. Then, for all $x>0, t \geq 0$ and $\Lambda \in \mathcal{F}_{t}^{(e)}$,

$$
\lim _{s \rightarrow \infty} \mathbb{P}_{x}^{(e)}\left(\Lambda \mid \bar{K}_{u}>0,0 \leq u \leq s\right)=\mathbb{P}_{x}^{(e), \uparrow}(\Lambda)
$$

The following inequality is also important for our purposes. Recall that $\hat{\kappa}$ denotes the Laplace exponent of the descending ladder process (see identity (2.2)) and that $\tau_{0}^{-}=\inf \left\{s \geq 0: \bar{K}_{s} \leq 0\right\}$.

Lemma 2.2. For $x>0$, we have

$$
\mathbb{P}_{x}^{(e)}\left(\tau_{0}^{-}>t\right) \leq 2 e \widehat{\kappa}(1 / t, 0) \widehat{V}(x), \quad \text { for } \quad t>0 .
$$

Proof. We first observe that the following series of inequalities holds for $q, t>0$,

$$
\frac{t}{2} e^{-q t} \mathbb{P}_{x}^{(e)}\left(\tau_{0}^{-}>t\right) \leq \int_{t / 2}^{t} e^{-q s} \mathbb{P}_{x}^{(e)}\left(\tau_{0}^{-}>s\right) \mathrm{d} s \leq \int_{0}^{\infty} e^{-q s} \mathbb{P}_{x}^{(e)}\left(\tau_{0}^{-}>s\right) \mathrm{d} s .
$$

From the Wiener-Hopf factorization (2.4), we have

$$
\mathbb{E}^{(e)}\left[e^{\theta I_{\mathbf{e}_{q}}}\right]=\frac{\widehat{\kappa}(q, 0)}{\widehat{\kappa}(q, \theta)}
$$


where $\mathbf{e}_{q}$ is an exponential random variable with parameter $q>0$, which is independent of $\bar{K}$. Hence, by a classical identity on tail distribution using Fubini's theorem, we deduce

$$
\begin{aligned}
\frac{\widehat{\kappa}(q, 0)}{\widehat{\kappa}(q, \theta)} & =\int_{0}^{\infty} e^{-\theta x} \mathbb{P}^{(e)}\left(-I_{\mathbf{e}_{q}} \in \mathrm{d} x\right) \\
& =\theta \int_{0}^{\infty} e^{-\theta x} \mathbb{P}^{(e)}\left(I_{\mathbf{e}_{q}}>-x\right) \mathrm{d} x=\theta \int_{0}^{\infty} e^{-\theta x} \mathbb{P}_{x}^{(e)}\left(\tau_{0}^{-}>\mathbf{e}_{q}\right) \mathrm{d} x .
\end{aligned}
$$

Next, for every $q>0$, we consider the function given by

$$
\widehat{V}^{(q)}(x):=\mathbb{E}^{(e)}\left[\int_{0}^{\infty} e^{-q \widehat{L}_{t}^{-1}} \mathbf{1}_{\left\{\widehat{H}_{t} \leq x\right\}} \mathrm{d} t\right]
$$

Performing a straightforward computation and using identity (2.2), we deduce

$$
\theta \int_{0}^{\infty} e^{-\theta x} \widehat{V}^{(q)}(x) \mathrm{d} x=\mathbb{E}^{(e)}\left[\int_{0}^{\infty} \exp \left\{-q \widehat{L}_{t}^{-1}-\theta \widehat{H}_{t}\right\} \mathrm{d} t\right]=\frac{1}{\widehat{\kappa}(q, \theta)} .
$$

The latter implies

$$
q \int_{0}^{\infty} e^{-q s} \mathbb{P}_{x}^{(e)}\left(\tau_{0}^{-}>s\right) \mathrm{d} s=\widehat{\kappa}(q, 0) \widehat{V}^{(q)}(x)
$$

We thus deduce for $t, q>0$, that

$$
\frac{t}{2} e^{-q t} \mathbb{P}_{x}^{(e)}\left(\tau_{0}^{-}>t\right) \leq \frac{\widehat{\kappa}(q, 0)}{q} \widehat{V}^{(q)}(x) \leq \frac{\widehat{\kappa}(q, 0)}{q} \widehat{V}(x) .
$$

Taking $q=1 / t$ yields (2.10), and completes the proof.

\section{CSBP IN A CONDITIONED RANDOM ENVIRONMENT}

\subsection{Definition and first properties}

Similarly to the definition of Lévy processes conditioned to stay positive [14] and following a similar strategy as in the discrete framework in Afanasyev et al. [2], we would like to introduce a CSBP in a Lévy environment conditioned to stay positive as a Doob- $h$ transform. In order to do so, we first observe that $\left(\widehat{V}\left(\bar{K}_{t}\right) \mathbf{1}_{\left\{I_{t} \geq 0\right\}}, t \geq 0\right)$ is also a martingale with respect to $\left(\mathcal{F}_{t}\right)_{t \geq 0}$, under $\mathbb{P}$. This result is more or less clear since it is a martingale under $\mathbb{P}^{(e)}$. Nonetheless we provide its proof for the sake of completeness.

Recall that $\mathbb{P}_{(z, x)}$ (resp. $\mathbb{E}_{(z, x)}$ its expectation) denotes the law of the couple $(Z, \bar{K})$ started from $(z, x)$ where $z, x>0$, under $\mathbb{P}$.

Lemma 3.1. Let us assume that $z, x>0$. The process $\left(\widehat{V}\left(\bar{K}_{t}\right) \mathbf{1}_{\left\{I_{t} \geq 0\right\}}, t \geq 0\right)$ is a martingale with respect to $\left(\mathcal{F}_{t}\right)_{t \geq 0}$, under $\mathbb{P}_{(z, x)}$.

Proof. Let $s \geq 0$ and $A \in \mathcal{F}_{s}$. We first claim that $\mathbb{P}(A \mid K)$ is a $\mathcal{F}_{s}^{(e)}$-measurable r.v. Indeed, since the family of sets

$$
\mathcal{C}_{s}=\left\{F_{b} \times F_{e}: F_{b} \in \mathcal{F}_{s}^{(b)}, F_{e} \in \mathcal{F}_{s}^{(e)}\right\}
$$


is a $\pi$-system that generates $\mathcal{F}_{s}$, we deduce that for any $D \in \mathcal{C}_{s}$ such that $D=B \times C$ with $B \in \mathcal{F}_{s}^{(b)}$ and $C \in \mathcal{F}_{s}^{(e)}$, the following identity holds

$$
\mathbb{P}(D \mid K)=\mathbf{1}_{C} \mathbb{P}(B \mid K)=\mathbf{1}_{C} \mathbb{P}^{(b)}(B),
$$

where in the last identity we have used that $B$ is independent of the environment and that $\mathbb{P}^{(b)}$ is the projection of $\mathbb{P}$ on $\Omega^{(b)}$. A monotone class argument allows us to conclude our claim.

Next, we assume $s \leq t$ and take $A \in \mathcal{F}_{s}$. By conditioning on the environment and recalling that $\mathbb{P}^{(e)}$ is the projection of $\mathbb{P}$ on $\Omega^{(e)}$, we observe

$$
\begin{aligned}
\mathbb{E}_{(z, x)}\left[\widehat{V}\left(\bar{K}_{t}\right) \mathbf{1}_{\left\{I_{t}>0\right\}} \mathbf{1}_{A}\right] & =\mathbb{E}_{(z, x)}\left[\widehat{V}\left(\bar{K}_{t}\right) \mathbf{1}_{\left\{I_{t}>0\right\}} \mathbb{P}_{(z, x)}(A \mid K)\right] \\
& =\mathbb{E}_{x}^{(e)}\left[\widehat{V}\left(\bar{K}_{t}\right) \mathbf{1}_{\left\{I_{t}>0\right\}} \mathbb{P}_{(z, x)}(A \mid K)\right]
\end{aligned}
$$

Let us now introduce the process $\widetilde{K}$ via $\widetilde{K}_{u}:=\bar{K}_{u+s}-\bar{K}_{s}$, for $u \geq 0$, which is independent of $\mathcal{F}_{s}^{(e)}$ and has the same law as $\bar{K}$. We also define its running infimum up to time $t$ by $\widetilde{I}_{t}$, i.e.

$$
\widetilde{I}_{t}=\inf _{0 \leq u \leq t} \widetilde{K}_{u}
$$

By taking $\mathfrak{P}$ a $\mathcal{F}_{s}^{(e)}$-measurable random variable, we deduce by conditioning on $\mathcal{F}_{s}^{(e)}$ and from identity $(2.8)$, that

$$
\begin{aligned}
\mathbb{E}_{x}^{(e)}\left[\widehat{V}\left(\bar{K}_{t}\right) \mathbf{1}_{\left\{I_{t}>0\right\}} \mathfrak{P}\right] & =\mathbb{E}_{x}^{(e)}\left[\widehat{V}\left(\bar{K}_{s}+K_{t-s}\right) \mathbf{1}_{\left\{\widetilde{I}_{t-s}+\bar{K}_{s}>0\right\}} \mathbf{1}_{\left\{I_{s}>0\right\}} \mathfrak{P}\right] \\
& =\mathbb{E}_{x}^{(e)}\left[\mathfrak{P} \mathbf{1}_{\left\{I_{s}>0\right\}} \mathbb{E}_{\bar{K}_{s}}^{(e)}\left[\widehat{V}\left(\widetilde{K}_{t-s}\right) \mathbf{1}_{\left\{\widetilde{I}_{t-s}>0\right\}}\right]\right] \\
& =\mathbb{E}_{x}^{(e)}\left[\mathfrak{P} \mathbf{1}_{\left\{I_{s}>0\right\}} \widehat{V}\left(\bar{K}_{s}\right)\right] .
\end{aligned}
$$

Putting all pieces together, we obtain

$$
\begin{aligned}
\mathbb{E}_{(z, x)}\left[\widehat{V}\left(\bar{K}_{t}\right) \mathbf{1}_{\left\{I_{t}>0\right\}} \mathbf{1}_{A}\right] & =\mathbb{E}_{x}^{(e)}\left[\widehat{V}\left(\bar{K}_{s}\right) \mathbf{1}_{\left\{I_{s}>0\right\}} \mathbb{P}_{(z, x)}(A \mid K)\right] \\
& =\mathbb{E}_{(z, x)}\left[\widehat{V}\left(\bar{K}_{s}\right) \mathbf{1}_{\left\{I_{s}>0\right\}} \mathbb{P}_{(z, x)}(A \mid K)\right] \\
& =\mathbb{E}_{(z, x)}\left[\widehat{V}\left(\bar{K}_{s}\right) \mathbf{1}_{\left\{I_{s}>0\right\}} \mathbf{1}_{A}\right]
\end{aligned}
$$

which allows us to conclude that the process $\left(\widehat{V}\left(\bar{K}_{t}\right) \mathbf{1}_{\left\{I_{t}>0\right\}}, t \geq 0\right)$ is a martingale with respect to $\left(\mathcal{F}_{t}\right)_{t \geq 0}$, under $\mathbb{P}_{(z, x)}$.

From the previous result, we construct the law of a CSBP in a Lévy environment conditioned to stay positive as a Doob- $h$ transform. To be more precise, for $\Lambda \in \mathcal{F}_{t}$ and $x, z>0$, we define

$$
\mathbb{P}_{(z, x)}^{\uparrow}(\Lambda):=\frac{1}{\widehat{V}(x)} \mathbb{E}_{(z, x)}\left[\widehat{V}\left(\bar{K}_{t}\right) \mathbf{1}_{\left\{I_{t}>0\right\}} \mathbf{1}_{\Lambda}\right]
$$

Similarly as in Lemma 2.1, the term Lévy environment conditioned to stay positive in definition (3.1) is justified from the following convergence result, which is crucial to prove Theorem 1.2. 
Lemma 3.2. Assume that Spitzer's condition $(\mathbf{H 1})$ holds and let $z, x>0$. For $t \geq 0$ and $\Lambda \in \mathcal{F}_{t}$, we have

$$
\lim _{s \rightarrow \infty} \mathbb{P}_{(z, x)}\left(\Lambda \mid \bar{K}_{u}>0,0 \leq u \leq s\right)=\mathbb{P}_{(z, x)}^{\uparrow}(\Lambda)
$$

Moreover if $\left(G_{t}, t \geq 0\right)$ is a uniformly bounded process which is adapted to $\left(\mathcal{F}_{t}\right)_{t \geq 0}$ and such that it converges to $G_{\infty}$, as $t \rightarrow \infty, \mathbb{P}_{(z, x)}^{\uparrow}$-almost surely, then

$$
\lim _{t \rightarrow \infty} \mathbb{P}_{(z, x)}\left[G_{t} \mid \bar{K}_{u}>0,0 \leq u \leq t\right]=\mathbb{P}_{(z, x)}^{\uparrow}\left[G_{\infty}\right]
$$

Proof. We proceed similarly as in Proposition 1 in [14]. Let $h, t \geq 0$ and take $\Lambda \in \mathcal{F}_{t}$. Then from the Markov property at time $t$, we obtain

$$
\mathbb{P}_{(z, x)}\left(\Lambda \mid I_{t+h}>0\right)=\mathbb{E}_{(z, x)}\left[\mathbf{1}_{\Lambda} \frac{\mathbb{P}_{\bar{K}}^{(e)}\left(I_{h}>0\right)}{\mathbb{P}_{x}^{(e)}\left(I_{t+h}>0\right)} \mathbf{1}_{\left\{I_{t}>0\right\}}\right]
$$

From inequality (2.10), we see

$$
\frac{\mathbb{P}_{\bar{K}}^{(e)}\left(I_{h}>0\right)}{\mathbb{P}_{x}^{(e)}\left(I_{t+h}>0\right)} \mathbf{1}_{\left\{I_{t}>0\right\}} \leq 2 e \frac{\widehat{\kappa}\left(h^{-1}, 0\right)}{\mathbb{P}_{x}^{(e)}\left(\tau_{0}^{-}>t+h\right)} \widehat{V}\left(\bar{K}_{t}\right) \mathbf{1}_{\left\{I_{t}>0\right\}} .
$$

On the other hand from Spitzer's condition, we know that $\widehat{\kappa}(\cdot, 0)$ is regularly varying at $0+$ with index $1-\rho$ and $\mathbb{P}_{x}^{(e)}\left(\tau_{0}^{-}>\cdot\right)$ is also regularly varying with index $\rho-1$ at $\infty$. Moreover, there is a slowly varying function $\ell(\cdot)$ at $\infty$ such that

$$
\widehat{\kappa}(q, 0) \sim \frac{\Gamma(1+\rho)}{\rho} \ell(1 / q) q^{1-\rho}, \quad \text { as } \quad q \rightarrow 0
$$

and

$$
\mathbb{P}_{x}^{(e)}\left(\tau_{0}^{-}>t\right) \sim \widehat{V}(x) t^{\rho-1} \ell(t), \quad \text { as } \quad t \rightarrow \infty
$$

see for instance the proof of Theorem VI.18 in [7]. Therefore from Potter's Theorem (see Thm. 1.5.6 in Bingham et al. [10]) for any $C_{2}>1$ and $\delta>0$ there exists $M$ such that for $h \geq M$,

$$
\frac{\mathbb{P}_{\bar{K}}^{(e)}\left(I_{h}>0\right)}{\mathbb{P}_{x}^{(e)}\left(I_{t+h}>0\right)} \mathbf{1}_{\left\{I_{t}>0\right\}} \leq C(\rho)\left(1+\frac{t}{M}\right)^{1-\rho+\delta} \frac{\widehat{V}\left(\bar{K}_{t}\right)}{\widehat{V}(x)} \mathbf{1}_{\left\{I_{t}>0\right\}},
$$

with

$$
C(\rho)=\frac{2 e \Gamma(1+\rho)}{\rho} C_{2}
$$

Since $\mathbb{E}_{(z, x)}\left[\widehat{V}\left(\bar{K}_{t}\right) \mathbf{1}_{\left\{I_{t}>0\right\}}\right]=\widehat{V}(x)$ and $\widehat{V}$ is finite, we may apply Lebesgue's theorem of dominated convergence on the right-hand side of (3.2) when $h$ goes to $\infty$. We conclude from the asymptotic (1.8) and the definition of (3.1). 
For the second part of our statement, we use similar arguments as those used in Lemma 2.5 in [2]. We let $s \leq t$ and $\gamma \in(1,2]$ and apply the Markov property at time $t$ and inequality (2.10), to deduce that

$$
\begin{aligned}
\left|\mathbb{E}_{(z, x)}\left[G_{t}-G_{s} \mid I_{\gamma t}>0\right]\right| & \leq \mathbb{E}_{(z, x)}\left[\left|G_{t}-G_{s}\right| \frac{\mathbb{P}_{\bar{K}}^{(e)}\left(I_{(\gamma-1) t}>0\right)}{\mathbb{P}_{x}^{(e)}\left(I_{\gamma t}>0\right)} \mathbf{1}_{\left\{I_{t}>0\right\}}\right] \\
& \leq 2 e \frac{\widehat{\kappa}\left(\frac{1}{(\gamma-1) t}, 0\right)}{\mathbb{P}_{x}^{(e)}\left(\tau_{0}^{-}>\gamma t\right)} \mathbb{E}_{(z, x)}\left[\left|G_{t}-G_{s}\right| \widehat{V}\left(\bar{K}_{t}\right) \mathbf{1}_{\left\{I_{t}>0\right\}}\right] \\
& =2 e \frac{\widehat{\kappa}\left(\frac{1}{(\gamma-1) t}, 0\right) \widehat{V}(x)}{\mathbb{P}_{x}^{(e)}\left(\tau_{0}^{-}>\gamma t\right)} \mathbb{E}_{(z, x)}^{\uparrow}\left[\left|G_{t}-G_{s}\right|\right] .
\end{aligned}
$$

Again Potter's Theorem (see Thm. 1.5.6 in Bingham et al. [10]) guarantees that for any $C_{2}>1$ and $\delta>0$ there exists $M$ such that for $t \geq M$,

$$
\begin{aligned}
\mid \mathbb{E}_{(z, x)}\left[G_{t}-G_{s} \mid I_{\gamma t}\right. & >0] \mid \\
& \leq C(\rho) \max \left\{\left(\frac{\gamma}{\gamma-1}\right)^{\delta+1-\rho},\left(\frac{\gamma}{\gamma-1}\right)^{-\delta+1-\rho}\right\} \mathbb{E}_{(z, x)}^{\uparrow}\left[\left|G_{t}-G_{s}\right|\right],
\end{aligned}
$$

and $C(\rho)$ is defined in (3.4).

Let $\varepsilon>0$. As $\left(G_{t}, t \geq 0\right)$ is a uniformly bounded process which converges to $G_{\infty}$, as $t \rightarrow \infty, \mathbb{P}_{(z, x)}^{\uparrow}$-almost surely, there exists $A_{\varepsilon}>0$ such that for any $A_{\varepsilon} \leq s \leq t$,

$$
C(\rho) \max \left\{\left(\frac{\gamma}{\gamma-1}\right)^{\delta+1-\rho},\left(\frac{\gamma}{\gamma-1}\right)^{-\delta+1-\rho}\right\} \mathbb{E}_{(z, x)}^{\uparrow}\left[\left|G_{t}-G_{s}\right|\right] \leq \varepsilon
$$

and

$$
\left|\mathbb{E}_{(z, x)}^{\uparrow}\left[G_{s}\right]-\mathbb{E}_{(z, x)}^{\uparrow}\left[G_{\infty}\right]\right| \leq \varepsilon
$$

Hence for any $s \geq A_{\varepsilon}$, letting $t$ go to infinity and applying the first statement of this lemma, we get

$$
\limsup _{t \rightarrow \infty}\left|\frac{\mathbb{E}_{(z, x)}\left[G_{t} \mathbf{1}_{\left\{I_{\gamma t}>0\right\}}\right]}{\mathbb{P}_{x}^{(e)}\left(I_{\gamma t}>0\right)}-\mathbb{E}_{(z, x)}^{\uparrow}\left[G_{s}\right]\right| \leq \varepsilon
$$

Adding (3.5), we get

$$
\mathbb{E}_{(z, x)}\left[G_{t} \mathbf{1}_{\left\{I_{\gamma t}>0\right\}}\right]=\left(\mathbb{E}_{(z, x)}^{\uparrow}\left[G_{\infty}\right]+o(1)\right) \mathbb{P}_{x}^{(e)}\left(I_{\gamma t}>0\right)
$$

Thus, there exists a constant $C_{3}>0$ such that

$$
\begin{aligned}
\left|\mathbb{E}_{(z, x)}\left[G_{t} \mathbf{1}_{\left\{I_{t}>0\right\}}\right]-\mathbb{E}_{(z, x)}^{\uparrow}\left[G_{\infty}\right] \mathbb{P}_{x}^{(e)}\left(I_{t}>0\right)\right| & \leq C_{3} \mathbb{P}_{x}^{(e)}\left(I_{t}>0, I_{\gamma t} \leq 0\right) \\
& +\left|\mathbb{E}_{(z, x)}\left[G_{t} \mathbf{1}_{\left\{I_{\gamma t}>0\right\}}\right]-\mathbb{E}_{(z, x)}^{\uparrow}\left[G_{\infty}\right] \mathbb{P}_{x}^{(e)}\left(I_{\gamma t}>0\right)\right| \\
& \leq\left(o(1)+c\left(1-\gamma^{\rho-1}\right)\right) \mathbb{P}_{x}^{(e)}\left(I_{t}>0\right),
\end{aligned}
$$


where we applied the asymptotic in (1.9) for the second inequality. Note that since $\ell$ in (1.9) is slowly varying and $\gamma \in(1,2]$, we can choose $c$ independent from $\gamma$ (see again Thm. 1.5.6 in Bingham et al. [10]). Since the choice of $\gamma$ on $(1,2]$ was arbitrary, we finally obtain

$$
\mathbb{E}_{(z, x)}\left[G_{t} \mathbf{1}_{\left\{I_{t}>0\right\}}\right]-\mathbb{E}_{(z, x)}^{\uparrow}\left[G_{\infty}\right] \mathbb{P}_{x}^{(e)}\left(I_{t}>0\right)=o\left(\mathbb{P}_{x}^{(e)}\left(I_{t}>0\right)\right)
$$

which completes the proof.

\subsection{Non-absorption}

In this section, we are interested in the event of survival of the process $Z$ under the conditioned environment. To estimate the latter, we first compute the probability of the event of extinction at a given time, under the conditioned environment, and then we will observe that such a probability is strictly positive if and only if Grey's condition (1.11) is fullfilled. It is important to note that the latter statement can be deduced directly from Theorem 4.1 in [19] but actually, in this case its proof is rather simple and for completeness we decide to include it.

Recall from Proposition 2 in [26] (or after the comments of Thm. 1), that there exists a functional $v_{t}(s, \lambda, \bar{K}$ ) which is the $\mathbb{P}^{(e)}$-a.s. unique solution of the backward differential equation given in (1.13) and satisfies

$$
\mathbb{E}_{(z, 0)}\left[\exp \left\{-\lambda e^{-x} Z_{t} e^{-\bar{K}_{t}}\right\} \mid \mathcal{F}_{t}^{(e)}\right]=\exp \left\{-z v_{t}\left(0, \lambda e^{-x}, \bar{K}\right)\right\} .
$$

A similar identity holds for CSBPs in a Lévy environment conditioned to stay positive as we see below.

Proposition 3.3. For $x, z>0$ and $\lambda \geq 0$, we have

$$
\mathbb{E}_{(z, x)}^{\uparrow}\left[e^{-\lambda Z_{t} e^{-\bar{K}_{t}}}\right]=\mathbb{E}_{x}^{(e), \uparrow}\left[e^{-z v_{t}\left(0, \lambda e^{-K_{0}}, \bar{K}-K_{0}\right)}\right]
$$

In particular,

$$
\mathbb{P}_{(z, x)}^{\uparrow}\left(Z_{t}=0\right)=\mathbb{E}_{x}^{(e), \uparrow}\left[e^{-z v_{t}\left(0, \infty, \bar{K}-K_{0}\right)}\right], \quad \text { for } \quad t>0
$$

which is strictly positive if and only if Grey's condition (1.11) is satisfied.

Proof. Let $x, z>0$. From the definition of CSBPs in a Lévy environment conditioned to stay positive (3.1), we deduce that for every non-negative $\lambda$,

$$
\begin{aligned}
\mathbb{E}_{(z, x)}^{\uparrow}\left[e^{-\lambda Z_{t} e^{-\bar{K}_{t}}}\right] & =\frac{1}{\widehat{V}(x)} \mathbb{E}_{(z, x)}\left[\widehat{V}\left(\bar{K}_{t}\right) e^{-\lambda Z_{t} e^{-\bar{K}_{t}}} \mathbf{1}_{\left\{I_{t}>0\right\}}\right] \\
& =\frac{1}{\widehat{V}(x)} \mathbb{E}_{(z, 0)}\left[\widehat{V}\left(\bar{K}_{t}+x\right) e^{-\lambda e^{-x} Z_{t} e^{-\bar{K}_{t}}} \mathbf{1}_{\left\{I_{t}>-x\right\}}\right] \\
& =\frac{1}{\widehat{V}(x)} \mathbb{E}_{(z, 0)}\left[\widehat{V}\left(\bar{K}_{t}+x\right) \mathbf{1}_{\left\{I_{t}>-x\right\}} \mathbb{E}_{(z, 0)}\left[e^{-\lambda e^{-x} Z_{t} e^{-\bar{K}_{t}}} \mid \mathcal{F}_{t}^{(e)}\right]\right] \\
& =\frac{1}{\widehat{V}(x)} \mathbb{E}_{(z, 0)}\left[\widehat{V}\left(\bar{K}_{t}+x\right) \mathbf{1}_{\left\{I_{t}>-x\right\}} e^{-v_{t}\left(0, \lambda e^{-x}, \bar{K}\right)}\right] \\
& =\frac{1}{\widehat{V}(x)} \mathbb{E}_{(z, x)}\left[\widehat{V}\left(\bar{K}_{t}\right) \mathbf{1}_{\left\{I_{t}>0\right\}} e^{-v_{t}\left(0, \lambda e^{-K_{0}}, \bar{K}-K_{0}\right)}\right] \\
& =\mathbb{E}_{x}^{(e), \uparrow}\left[e^{-z v_{t}\left(0, \lambda e^{-K_{0}}, \bar{K}-K_{0}\right)}\right] .
\end{aligned}
$$


By letting $\lambda$ go to infinity, we get

$$
\mathbb{P}_{(z, x)}^{\uparrow}\left(Z_{t}=0\right)=\mathbb{E}_{x}^{(e), \uparrow}\left[e^{-z v_{t}\left(0, \infty, \bar{K}-K_{0}\right)}\right]
$$

From the previous identity, it is clear that

$$
0<\mathbb{P}_{(z, x)}^{\uparrow}\left(Z_{t}=0\right) \quad \text { if and only if } \quad \mathbb{P}_{x}^{(e), \uparrow}\left(v_{t}\left(0, \infty, \bar{K}-K_{0}\right)<\infty\right)>0
$$

Therefore, in order to deduce the last statement it is enough to show that Grey's condition (1.11) is necessary and sufficient for $\mathbb{P}_{x}^{(e), \uparrow}\left(v_{t}\left(0, \infty, \bar{K}-K_{0}\right)<\infty\right)>0$.

We first observe from the Wiener-Hopf factorisation (2.4) applied to the spectrally positive Lévy process associated to the branching mechanism $\psi_{0}$, that there exists a non decreasing function $\Phi$ (which is associated to its ascending ladder height) satisfying,

$$
\psi_{0}(\lambda)=\lambda \Phi(\lambda) \quad \text { for } \quad \lambda \geq 0
$$

More precisely, from (1.2), (1.10) and integration by parts, we have

$$
\begin{aligned}
\Phi(\lambda) & =\gamma^{2} \lambda+\int_{(0, \infty)} \frac{e^{-\lambda x}-1+\lambda x}{\lambda} \mu(\mathrm{d} x) \\
& =\gamma^{2} \lambda+\int_{(0, \infty)}\left(1-e^{-\lambda x}\right) \bar{\mu}(x) \mathrm{d} x,
\end{aligned}
$$

where $\bar{\mu}(x):=\mu((x, \infty))$. Since $\Phi$ is the Laplace exponent of a subordinator, it is well-known that for any $\lambda>0$ and $k>1$, we have $\Phi(\lambda) \leq k \Phi(\lambda / k)$ (see for instance the proof of Prop. III.1 in [7]). In particular, from (1.13) and under the event that $\left\{t<\tau_{-x}^{-}\right\}$, we have

$$
\begin{aligned}
\frac{\partial}{\partial s} v_{t}\left(s, \lambda e^{-x}, \bar{K}-x\right) & =e^{\bar{K}_{s}-x} \psi_{0}\left(e^{-\bar{K}_{s}+x} v_{t}\left(s, \lambda e^{-x}, \bar{K}-x\right)\right) \\
& =v_{t}\left(s, \lambda e^{-x}, \bar{K}-x\right) \Phi\left(e^{-\bar{K}_{s}+x} v_{t}\left(s, \lambda e^{-x}, \bar{K}-x\right)\right) \\
& \geq e^{-\bar{K}_{s}+x} \psi_{0}\left(v_{t}\left(s, \lambda e^{-x}, \bar{K}-x\right)\right)
\end{aligned}
$$

This entails

$$
\int_{v_{t}\left(0, \lambda e^{-x}, \bar{K}-x\right)}^{\lambda e^{-x}} \frac{\mathrm{d} u}{\psi_{0}(u)} \geq \int_{0}^{t} e^{-\bar{K}_{s}+x} \mathrm{~d} s .
$$

Assuming that (1.11) holds, we deduce

$$
\int_{v_{t}(0, \infty, \bar{K}-x)}^{\infty} \frac{\mathrm{d} u}{\psi_{0}(u)} \geq \int_{0}^{t} e^{-\bar{K}_{s}+x} \mathrm{~d} s,
$$

which clearly implies that $v_{t}\left(0, \infty, \bar{K}-K_{0}\right)<\infty$ with positive probability under $\mathbb{P}_{x}^{(e), \uparrow}$. 
Next, we assume that $\mathbb{P}_{x}^{(e),}\left(v_{t}\left(0, \infty, \bar{K}-K_{0}\right)<\infty\right)>0$. Since $\psi_{0}$ is non-decreasing, we deduce, under the event that $\left\{t<\tau_{-x}^{-}\right\}$, that

$$
\begin{aligned}
\frac{\partial}{\partial s} v_{t}\left(s, \lambda e^{-x}, \bar{K}-x\right) & =e^{\bar{K}_{s}-x} \psi_{0}\left(e^{-\bar{K}_{s}+x} v_{t}\left(s, \lambda e^{-x}, \bar{K}-x\right)\right) \\
& \leq e^{\bar{K}_{s}-x} \psi_{0}\left(v_{t}\left(s, \lambda e^{-x}, \bar{K}-x\right)\right)
\end{aligned}
$$

which implies

$$
\int_{v_{t}\left(0, e^{-x}, \bar{K}-x\right)}^{\lambda e^{-x}} \frac{\mathrm{d} u}{\psi_{0}(u)} \leq \int_{0}^{t} e^{\bar{K}_{s}-x} \mathrm{~d} s
$$

Therefore, by letting $\lambda$ goes to $\infty$, we have

$$
\int_{v_{t}(0, \infty, \bar{K}-x)}^{\infty} \frac{\mathrm{d} u}{\psi_{0}(u)} \leq \int_{0}^{t} e^{\bar{K}_{s}-x} \mathrm{~d} s
$$

with positive probability under $\mathbb{P}_{x}^{(e), \uparrow}$. It implies that Grey's condition (1.11) holds and completes the proof.

Actually, from Grey's condition (1.11) and inequality (3.9), we can deduce a nice lower bound for the probability of extinction. Indeed, let us introduce

$$
f(t):=\int_{t}^{\infty} \frac{\mathrm{d} u}{\psi_{0}(u)}, \quad \text { for } \quad t>0
$$

and note that the function $f:(0, \infty) \rightarrow(0, \infty)$ is a decreasing bijection and thus its inverse exists. We denote this inverse by $\varphi$. Therefore from (3.9), we get

$$
v_{t}(0, \infty, \bar{K}-x) \leq \varphi\left(\int_{0}^{t} e^{-\bar{K}_{s}+x} \mathrm{~d} s\right)
$$

In other words,

$$
\mathbb{E}_{x}^{(e), \uparrow}\left[\exp \left\{-z \varphi\left(\int_{0}^{t} e_{s}^{-\bar{K}}\right) \mathrm{d} s\right\}\right] \leq \mathbb{E}_{x}^{(e), \uparrow}\left[e^{-z v_{t}\left(0, \infty, \bar{K}-K_{0}\right)}\right]
$$

implying

$$
0<\mathbb{E}_{x}^{(e), \uparrow}\left[\exp \left\{-z \varphi\left(\int_{0}^{\infty} e^{-\bar{K}_{s}}\right) \mathrm{d} s\right\}\right] \leq \lim _{t \rightarrow \infty} \mathbb{P}_{(z, x)}^{\uparrow}\left(Z_{t}=0\right)
$$

since $\varphi$ is non-increasing and

$$
\int_{0}^{\infty} e^{-\bar{K}_{s}} \mathrm{~d} s<\infty, \quad \mathbb{P}_{x}^{(e), \uparrow-\text { a.s. }}
$$


The claim in (3.10) follows from the following argument. From Theorem VI.20 in Bertoin [7], we observe

$$
\begin{aligned}
& \mathbb{E}_{x}^{(e), \uparrow}\left[\int_{0}^{\infty} e^{-\bar{K}_{s}} \mathrm{~d} s\right]=\int_{0}^{\infty} \mathbb{E}_{x}^{(e), \uparrow}\left[e^{-\bar{K}_{s}}\right] \mathrm{d} s \\
& =\int_{0}^{\infty} \frac{1}{\widehat{V}(x)} \mathbb{E}_{x}^{(e)}\left[e^{-\bar{K}_{s}} \widehat{V}\left(\bar{K}_{s}\right) \mathbf{1}_{\left\{I_{s}>0\right\}}\right] \mathrm{d} s \\
& =\frac{1}{\widehat{V}(x)} \mathbb{E}_{x}^{(e)}\left[\int_{0}^{\tau_{0}^{-}} e^{-\bar{K}_{s}} \widehat{V}\left(\bar{K}_{s}\right) \mathrm{d} s\right] \\
& =\frac{\mathbf{c}}{\widehat{V}(x)} \int_{[0, \infty)} V(\mathrm{~d} y) \int_{[0, x]} \widehat{V}(\mathrm{~d} z) e^{-x-y+z} \widehat{V}(x+y-z),
\end{aligned}
$$

where we recall that $V$ denotes the renewal measure associated to the ascending ladder height and $\mathbf{c}$ is a constant that only depends on the normalisation of the local times $L$ and $\widehat{L}$. For the sake of simplicity we take $\mathbf{c}=1$. Thus, since $\widehat{V}$ is increasing we have

$$
\mathbb{E}_{x}^{(e), \uparrow}\left[\int_{0}^{\infty} e^{-\bar{K}_{s}} \mathrm{~d} s\right] \leq \int_{[0, \infty)} V(\mathrm{~d} y) e^{-y} \widehat{V}(x+y)
$$

The latter integral is finite since $\widehat{V}$ satisfies $(2.5)$ and

$$
\int_{[0, \infty)} e^{-\theta x} V(\mathrm{~d} x)=\frac{1}{\kappa(0, \theta)}, \quad \text { for } \quad \theta>0
$$

which follows from the definition of $V$ (see (2.7)) and similar arguments as in (2.6). In other words the claim in (3.10) holds.

On the other hand, for our purposes, we are interested in conditions which guarantee that

$$
\lim _{t \rightarrow \infty} \mathbb{P}_{(z, x)}^{\uparrow}\left(Z_{t}=0\right)<1
$$

This problem is similar to determining when the probability of survival of a CSBP process in Lévy environments that drifts to $+\infty$, is positive. According to Proposition 2 in [25], the latter holds under a $x \log (x)$ moment condition on the measure $\mu$.

Assumption (H2) is very similar to the previous condition and implies that $Z$ has a positive probability to survive when living in a "favorable" environment, or in other words when the running infimum of the Lévy environment is positive.

Proposition 3.4. If condition (H2) holds then

$$
\lim _{t \rightarrow \infty} \mathbb{P}_{(z, x)}^{\uparrow}\left(Z_{t}>0\right)>0
$$

Proof. Let us assume that condition (H2) holds. We follow similar ideas as in the proof of Proposition 2 in [25]. First recall that the function $s \mapsto v_{t}\left(s, \lambda e^{-x}, \bar{K}-x\right)$ is non-decreasing on $[0, t]$ since $\psi_{0}$ is positive. Hence for any $s \in[0, t]$, we have

$$
v_{t}\left(s, \lambda e^{-x}, \bar{K}-x\right) \leq \lambda e^{-x}
$$


In particular, from (1.13) we have

$$
\begin{aligned}
\frac{\partial}{\partial s} v_{t}\left(s, \lambda e^{-x}, \bar{K}-x\right) & =e^{\bar{K}_{s}-x} \psi_{0}\left(e^{-\bar{K}_{s}+x} v_{t}\left(s, \lambda e^{-x}, \bar{K}-x\right)\right) \\
& =v_{t}\left(s, \lambda e^{-x}, \bar{K}-x\right) \Phi\left(e^{-\bar{K}_{s}+x} v_{t}\left(s, \lambda e^{-x}, \bar{K}-x\right)\right) \\
& \leq v_{t}\left(s, \lambda e^{-x}, \bar{K}-x\right) \Phi\left(e^{-\bar{K}_{s}} \lambda\right)
\end{aligned}
$$

as $\Phi$ is non-decreasing and $v_{t}\left(s, \lambda e^{-x}, \bar{K}-x\right)$ is non-decreasing with $s$ and equals $\lambda e^{-x}$ when $s=t$. This entails

$$
v_{t}\left(0, \lambda e^{-x}, \bar{K}-x\right) \geq \lambda e^{-x} \exp \left\{-\int_{0}^{t} \Phi\left(\lambda e^{-\bar{K}_{s}}\right) \mathrm{d} s\right\}
$$

Thus, for any $\lambda \geq 0$

$$
\begin{aligned}
\mathbb{P}_{(z, x)}^{\uparrow}\left(Z_{t}=0\right) & =\mathbb{E}_{x}^{(e), \uparrow}\left[e^{-z v_{t}(0, \infty, \bar{K}-x)}\right] \\
& =\frac{1}{\widehat{V}(x)} \mathbb{E}_{x}^{(e)}\left[\widehat{V}\left(\bar{K}_{t}\right) e^{-z v_{t}(0, \infty, \bar{K}-x)} \mathbf{1}_{\left\{t<\tau_{0}^{-}\right\}}\right] \\
& \leq \frac{1}{\widehat{V}(x)} \mathbb{E}_{x}^{(e)}\left[\widehat{V}\left(\bar{K}_{t}\right) e^{-z v_{t}\left(0, \lambda e^{-x}, \bar{K}-x\right)} \mathbf{1}_{\left\{t<\tau_{0}^{-}\right\}}\right] \\
& \leq \frac{1}{\widehat{V}(x)} \mathbb{E}_{x}^{(e)}\left[\widehat{V}\left(\bar{K}_{t}\right) e^{-z \lambda e^{-x} \exp \left\{-\int_{0}^{t} \Phi\left(\lambda e^{-\bar{K}_{s}}\right) \mathrm{d} s\right\}} \mathbf{1}_{\left\{t<\tau_{0}^{-}\right\}}\right] \\
& =\mathbb{E}_{x}^{(e), \uparrow}\left[e^{-z \lambda e^{-x} \exp \left\{-\int_{0}^{t} \Phi\left(\lambda e^{-\bar{K}_{s}}\right) \mathrm{d} s\right\}}\right]
\end{aligned}
$$

where we have used that $\lambda \mapsto v_{t}(0, \lambda, \bar{K}-x)$ is non-decreasing, see Proposition 2.2 in He et al. [19]. Hence, we have

$$
\lim _{t \rightarrow \infty} \mathbb{P}_{(z, x)}^{\uparrow}\left(Z_{t}=0\right) \leq \mathbb{E}_{x}^{(e), \uparrow}\left[e^{-z \lambda e^{-x} \exp \left\{-\int_{0}^{\infty} \Phi\left(\lambda e^{-\bar{K}_{s}}\right) \mathrm{d} s\right\}}\right]
$$

If

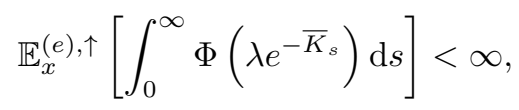

we get

$$
\exp \left\{-\int_{0}^{\infty} \Phi\left(\lambda e^{-\bar{K}_{s}}\right) \mathrm{d} s\right\}>0, \quad \mathbb{P}_{x}^{(e), \uparrow}-\text { a.s. }
$$

and $\lim _{t \rightarrow \infty} \mathbb{P}_{(z, x)}^{\uparrow}\left(Z_{t}=0\right)<1$. In other words, in order to deduce our result it is enough to show that (3.12) holds. We proceed similarly as in the proof of (3.10). From the definition of $\mathbb{P}_{x}^{(e), \uparrow}$ and Theorem VI.20 in 
Bertoin [7], we observe

$$
\begin{aligned}
& \mathbb{E}_{x}^{(e), \uparrow}\left[\int_{0}^{\infty} \Phi\left(\lambda e^{-\bar{K}_{s}}\right) \mathrm{d} s\right]=\frac{1}{\widehat{V}(x)} \mathbb{E}_{x}^{(e)}\left[\int_{0}^{\tau_{0}^{-}} \Phi\left(\lambda e^{-\bar{K}_{s}}\right) \widehat{V}\left(\bar{K}_{s}\right) \mathrm{d} s\right] \\
& =\frac{1}{\widehat{V}(x)} \int_{[0, \infty)} V(\mathrm{~d} y) \int_{[0, x]} \widehat{V}(\mathrm{~d} z) \Phi\left(\lambda e^{-x-y+z}\right) \widehat{V}(x+y-z) .
\end{aligned}
$$

Recalling the definition of $\Phi$ in (3.8) and observing that it is increasing, as well as the renewal function $\widehat{V}$, we obtain that

$$
\begin{aligned}
& \frac{1}{\widehat{V}(x)} \int_{[0, \infty)} V(\mathrm{~d} y) \int_{[0, x]} \widehat{V}(\mathrm{~d} z) \Phi\left(\lambda e^{-x-y+z}\right) \widehat{V}(x+y-z) \\
& \leq \int_{[0, \infty)} V(\mathrm{~d} y) \Phi\left(\lambda e^{-y}\right) \widehat{V}(x+y) \\
&=\gamma^{2} \lambda \int_{[0, \infty)} V(\mathrm{~d} y) e^{-y} \widehat{V}(x+y) \\
&+\int_{[0, \infty)} V(\mathrm{~d} y) \widehat{V}(x+y) \int_{(0, \infty)}\left(1-e^{-\lambda e^{-y} z}\right) \bar{\mu}(z) \mathrm{d} z .
\end{aligned}
$$

The first integral of the right-hand side is finite from identity (3.11) and since $\widehat{V}$ satisfies (2.5). For the second integral, we first rewrite

$$
\begin{aligned}
\int_{[0, \infty)} V(\mathrm{~d} y) & \widehat{V}(x+y) \int_{(0, \infty)}\left(1-e^{-\lambda e^{-y} z}\right) \bar{\mu}(z) \mathrm{d} z \\
& =\int_{(0, \infty)} \mathrm{d} z \bar{\mu}(z) \int_{[0, \infty)} V(\mathrm{~d} y)\left(1-e^{-\lambda e^{-y} z}\right) \widehat{V}(x+y) \\
& =\int_{(0, \infty)} \mathrm{d} z \bar{\mu}(z) g(z)
\end{aligned}
$$

with

$$
g(z):=\int_{[0, \infty)} V(\mathrm{~d} y)\left(1-e^{-\lambda e^{-y} z}\right) \widehat{V}(x+y) .
$$

In order to conclude our proof, we need to show that under condition (H2), the integral of $z \mapsto \bar{\mu}(z) g(z)$ is finite. In other words, we need to study the behaviour of $g(z)$ when $z$ is close to 0 and to $\infty$. With this aim in mind, we use that $\widehat{V}$ is subadditive and identity (3.11), as well as the following inequality,

$$
1-e^{-z} \leq 1 \wedge z
$$


which holds for every $z>0$. For $z$ small enough, and using inequality (2.5), we get

$$
\begin{aligned}
g(z) & \leq \lambda z \int_{[0,1)} V(\mathrm{~d} y) \widehat{V}(x+y) e^{-y}+\lambda z \int_{[1, \infty)} V(\mathrm{~d} y) \widehat{V}(x+y) e^{-y} \\
& \leq \lambda z\left(\widehat{V}(x+1) V(1)+C(x) \int_{[1, \infty)} V(\mathrm{~d} y) y e^{-y}\right) \\
& \leq C_{1}(x) \lambda z
\end{aligned}
$$

where $C(x)$ and $C_{1}(x)$ are two finite constants that only depend on $x$.

For $z$ large enough, we split the integral in (3.13) into three terms. To be more precise,

$$
\begin{aligned}
g(z) \leq & \int_{[0, \infty)} V(\mathrm{~d} y) \widehat{V}(x+y)\left(\lambda z e^{-y} \wedge 1\right) \\
\leq & \int_{[0,1)} V(\mathrm{~d} y) \widehat{V}(x+y)+\int_{[1,2 \ln (\lambda z))} V(\mathrm{~d} y) \widehat{V}(x+y) \\
& +\lambda z \int_{[2 \ln (\lambda z), \infty)} V(\mathrm{~d} y) \widehat{V}(x+y) e^{-y} .
\end{aligned}
$$

We study the three terms from above separately. First, it is clear that the first term satisfies

$$
\int_{[0,1)} V(\mathrm{~d} y) \widehat{V}(x+y) \leq V(1) \widehat{V}(x+1)
$$

For the third term, we use (2.5) and deduce

$$
\begin{aligned}
\lambda z \int_{2 \ln (\lambda z)}^{\infty} V(\mathrm{~d} y) \widehat{V}(x+y) e^{-y} & \leq C_{1} \lambda z e^{-2 \ln (\lambda z) / 2} \int_{2 \ln (\lambda z)}^{\infty} V(\mathrm{~d} y)(x+y) e^{-y / 2} \\
& \leq C_{1}(x+1) \int_{0}^{\infty} V(\mathrm{~d} y)(1+y) e^{-y / 2}
\end{aligned}
$$

where

$$
\int_{0}^{\infty} V(\mathrm{~d} y)(1+y) e^{-y / 2} \leq \sum_{i \geq 0} V([i, i+1))(1+i+1) e^{-i / 2} \leq C_{4} \sum_{i \geq 0}(i+2)^{2} e^{-i / 2}<\infty
$$

with $C_{4}>0$ such that

$$
V(x) \leq C_{4} x, \quad \text { for } \quad x \geq 0
$$

Finally,

$$
\int_{[1,2 \ln (\lambda z))} V(\mathrm{~d} y) \widehat{V}(x+y) \leq C(x) \int_{[1,2 \ln (\lambda z))} y V(\mathrm{~d} y) \leq C_{2}(x) \ln ^{2}(\lambda z),
$$

where $C(x)$ and $C_{2}(x)$ are two finite constants that only depend on $x$. Since condition (H2) holds, the proof of our result is now complete. 


\section{Proof of Theorem 1.2}

We have now collected all the necessary results to study the asymptotic behaviour of the extinction probability of $Z$. The proof of Theorem 1.2 follows from studying the event of survival at time $t,\left\{Z_{t}>0\right\}$ in three different situations that depend on the behaviour of the infimum of the environment. To be more precise, we split the survival event as follows: for $z, x>0$,

$$
\mathbb{P}_{(z, x)}\left(Z_{t}>0\right)=\mathbb{P}_{(z, x)}\left(Z_{t}>0, I_{t}>0\right)+\mathbb{P}_{(z, x)}\left(Z_{t}>0,-y<I_{t} \leq 0\right)+\mathbb{P}_{(z, x)}\left(Z_{t}>0, I_{t} \leq-y\right),
$$

where $y>0$ will be chosen later on. In other words, to deduce our result, we study such events separately for $t$ sufficiently large.

Our first result in this section concerns the first term in the right hand side of (4.1). It says that this is the leading term in (4.1).

Lemma 4.1. Assume that assumptions (H1) and (H2) hold. For $z, x>0$, there exists a positive constant $c(z, x)$ such that

$$
\mathbb{P}_{(z, x)}\left(Z_{t}>0, I_{t}>0\right) \sim c(z, x) \mathbb{P}_{x}^{(e)}\left(I_{t}>0\right) \sim c(z, x) \widehat{V}(x) t^{-(1-\rho)} \ell(t), \quad \text { as } \quad t \rightarrow \infty,
$$

where $\ell$ is a slowly varying function at $\infty$, introduced in (1.9).

Proof. Since $\mathbf{1}_{\left\{Z_{t}>0\right\}}$ converges to $\mathbf{1}_{\left\{\forall s \geq 0, Z_{s}>0\right\}}, \mathbb{P}_{(z, x)}^{\uparrow}$-almost surely, as $t$ goes to $\infty$, we can apply Lemma 3.2 and

$$
\begin{aligned}
\mathbb{P}_{(z, x)}\left(Z_{t}>0, I_{t}>0\right) & =\mathbb{P}_{(z, x)}\left(Z_{t}>0 \mid I_{t}>0\right) \mathbb{P}_{x}^{(e)}\left(I_{t}>0\right) \\
& \sim \mathbb{P}_{(z, x)}^{\uparrow}\left(\forall s \geq 0, Z_{s}>0\right) \mathbb{P}_{x}^{(e)}\left(I_{t}>0\right), \quad \text { as } \quad t \rightarrow \infty .
\end{aligned}
$$

From Proposition 3.4, we know that

$$
\mathbb{P}_{(z, x)}^{\uparrow}\left(\forall s \geq 0, Z_{s}>0\right)>0
$$

We conclude the proof by recalling the asymptotic behaviour in (1.9) to deduce the second equivalence.

We now prove that the last term in the right hand side of (4.1) is negligible for $y$ large enough, under assumptions (H1) and (H3).

Lemma 4.2. Let $\varepsilon, z, x>0, \delta \in(0,1)$ and assume that assumptions $(\mathbf{H 1})$ and (H3) hold. Then for $t$ and $y$ large enough, we have

$$
\mathbb{P}_{(z, x)}\left(Z_{t}>0, I_{t-\delta}<-y\right) \leq \varepsilon \mathbb{P}_{(z, x+y)}\left(Z_{t}>0, I_{t-\delta}>0\right)
$$

Proof. Recall that for $s \in[0, t]$, the functional $v_{t}(s, \lambda, \bar{K})$ is the $\mathbb{P}^{(e)}$-a.s. unique solution of the backward differential equation (1.13). We also recall that the quenched survival probability satisfies

$$
\mathbb{P}_{(z, x)}\left(Z_{t}>0 \mid \bar{K}\right)=1-e^{-z v_{t}(0, \infty, \bar{K}-x)}
$$

From assumption (H3) and definition (1.13), we obtain that for $s \leq t$ and $\lambda \geq 0$,

$$
\begin{aligned}
\frac{\partial}{\partial s} v_{t}\left(s, \lambda e^{-x}, \bar{K}-x\right) & \geq C e^{\bar{K}_{s}-x}\left(v_{t}\left(s, \lambda e^{-x}, \bar{K}-x\right) e^{-\bar{K}_{s}+x}\right)^{\beta+1} \\
& =C v_{t}^{\beta+1}\left(s, \lambda e^{-x}, \bar{K}-x\right) e^{-\beta\left(\bar{K}_{s}-x\right)}
\end{aligned}
$$


This yields

$$
\frac{1}{v_{t}^{\beta}\left(0, \lambda e^{-x}, \bar{K}-x\right)}-\frac{1}{\lambda^{\beta}} \geq \beta C \mathcal{I}_{t}(\beta(\bar{K}-x)),
$$

where

$$
\mathcal{I}_{t}(\beta(\bar{K}-x)):=\int_{0}^{t} e^{-\beta\left(\bar{K}_{s}-x\right)} \mathrm{d} s .
$$

Letting $\lambda$ go to $\infty$, we obtain

$$
v_{t}(0, \infty, \bar{K}-x) \leq\left(\beta C \mathcal{I}_{t}\left(\beta\left(\bar{K}_{s}-x\right)\right)\right)^{-1 / \beta} .
$$

Using (4.2) and (4.3), we get the following upper bound

$$
\begin{aligned}
\mathbb{P}_{(z, x)}\left(Z_{t}>0, I_{t-\delta}<-y\right) \leq \mathbb{E}_{x}^{(e)}\left[\left(1-e^{-z\left(\beta C \mathcal{I}_{t}(\beta(\bar{K}-x))\right)^{-1 / \beta}}\right)\right. & \left.\mathbf{1}_{\left\{I_{t-\delta}<-y\right\}}\right] \\
= & \mathbb{E}^{(e)}\left[\left(1-e^{-z\left(\beta C \mathcal{I}_{t}(\beta(\bar{K}))\right)^{-1 / \beta}}\right) \mathbf{1}_{\left\{I_{t-\delta}<-y-x\right\}}\right] .
\end{aligned}
$$

On the other hand, under assumption (H1), Theorems 2.18 and 2.20 in $[28]$ guarantee that for $q \in(0,1)$,

$$
\mathbb{E}^{(e)}\left[\mathcal{I}_{t}(\beta \bar{K})^{-q}\right]<\infty, \quad t>0,
$$

and for $F \in C_{b}\left(\mathbb{R}_{+}\right)$

$$
\lim _{t \rightarrow \infty} \frac{\mathbb{E}^{(e)}\left[\mathcal{I}_{t}(\beta \bar{K})^{-q} F\left(\mathcal{I}_{t}(\beta \bar{K})\right]\right.}{\widehat{\kappa}(1 / t, 0)}=\int_{0}^{\infty} F(x) \nu_{q, \rho}(\mathrm{d} x),
$$

where $\nu_{q, \rho}$ is a finite measure on $(0, \infty)$, see equation (2.46) in [28] for further details about $\nu_{q, \rho}$. Thus, by taking $F_{z}(x)=x^{q}\left(1-e^{-z C_{\beta} x^{-1 / \beta}}\right)$ with $C_{\beta}=(\beta C)^{-1 / \beta}$, we deduce

$$
\lim _{t \rightarrow \infty} \frac{\mathbb{E}^{(e)}\left[1-e^{-z C_{\beta}\left(\mathcal{I}_{t}(\beta(\bar{K}))\right)^{-1 / \beta}}\right]}{\widehat{\kappa}(1 / t, 0)}=\int_{0}^{\infty} x^{q}\left(1-e^{-z C_{\beta} x^{-1 / \beta}}\right) \nu_{q, \rho}(\mathrm{d} x)=: C_{\beta, q, \rho}(z),
$$

where the last notation has been introduced for the sake of readability. Hence, in particular from (3.3) we have

$$
\mathbb{E}^{(e)}\left[1-e^{-z C_{\beta}\left(\mathcal{I}_{t}(\beta(\bar{K}))\right)^{-1 / \beta}}\right] \sim \frac{\Gamma(1+\rho) C_{\beta, q, \rho}(z)}{\rho} t^{\rho-1} \ell(t), \quad \text { as } \quad t \rightarrow \infty,
$$

where $\ell$ is the slowly varying function at $\infty$ introduced in (3.3). The latter implies that there exists $t_{0}$ such that if $t \geq t_{0}$

$$
\mathbb{E}^{(e)}\left[1-e^{-z C_{\beta}\left(\mathcal{I}_{t}(\beta(\bar{K}))\right)^{-1 / \beta}}\right] \leq 2 \frac{\Gamma(1+\rho) C_{\beta, q, \rho}(z)}{\rho} t^{\rho-1} \ell(t)
$$

Next, we recall from (1.9) that

$$
\mathbb{P}^{(e)}\left(I_{t}>-y\right)=\mathbb{P}_{y}^{(e)}\left(I_{t}>0\right) \sim \widehat{V}(y) t^{\rho-1} \ell(t), \quad \text { as } \quad t \rightarrow \infty .
$$


On the other hand from Potter's Theorem (see Thm. 1.5.6 in Bingham et al. [10]), we deduce that for any $A>1$ and $\delta_{1}>0$ there exists $t_{1}:=t_{1}\left(A_{1}, \delta_{1}\right)$ such that for $s \geq h \geq t_{1}$,

$$
\frac{\mathbb{P}^{(e)}\left(I_{h}>-y\right)}{\mathbb{P}^{(e)}\left(I_{s}>-y\right)} \leq A\left(\frac{s}{h}\right)^{1-\rho+\delta_{1}}
$$

Let us fix $A>1$ and $\delta_{1}>0$ and introduce $\tau_{-y}=\inf \left\{t: \bar{K}_{t} \leq-y\right\}$, the first hitting time of $-y$ by $\bar{K}$. The previous inequality implies that for $s \geq h \geq t_{2}:=t_{0} \vee t_{1}$,

$$
\begin{aligned}
\mathbb{P}^{(e)}\left(h<\tau_{-y} \leq s\right) & =\mathbb{P}^{(e)}\left(I_{h}>-y\right)-\mathbb{P}^{(e)}\left(I_{s}>-y\right) \\
& =\mathbb{P}^{(e)}\left(I_{s}>-y\right)\left(\frac{\mathbb{P}^{(e)}\left(I_{h}>-y\right)}{\mathbb{P}^{(e)}\left(I_{s}>-y\right)}-1\right) \\
& \leq\left(A\left(\frac{s}{h}\right)^{1-\rho+\delta_{1}}-1\right) \mathbb{P}^{(e)}\left(I_{s}>-y\right) .
\end{aligned}
$$

For simplicity, we introduce the notation $\tilde{y}=y+x$. Hence from the property of independent increments of $\bar{K}$, we get the following sequence of inequalities, for $t \geq 3 t_{2}$,

$$
\begin{aligned}
\mathbb{E}^{(e)}\left[\left(1-e^{-z C_{\beta}\left(\mathcal{I}_{t}(\beta(\bar{K}))\right)^{-1 / \beta}}\right), \tau_{-\tilde{y}} \leq t-\delta\right] \\
\leq \mathbb{E}^{(e)}\left[\left(1-e^{-z C_{\beta}\left(\int_{\tau_{-\tilde{y}}}^{t} e^{-\beta \bar{K}_{s}} \mathrm{~d} s\right)^{-1 / \beta}}\right) \mathbf{1}_{\left\{\tau_{-\tilde{y}} \leq t-\delta\right\}}\right] \\
\leq \mathbb{E}^{(e)}\left[\left(1-\exp \left\{-z C_{\beta} e^{-\tilde{y}}\left(\int_{0}^{t-\tau_{-\tilde{y}}} e^{-\beta\left(\bar{K}_{\tau-\tilde{y}+u}-\bar{K}_{\tau-\tilde{y}}\right)} \mathrm{d} s\right)^{-1 / \beta}\right\}\right) \mathbf{1}_{\left\{\tau_{-\tilde{y}} \leq t-\delta\right\}}\right] \\
\leq \mathbb{E}^{(e)}\left[\left(1-e^{\left.\left.-z C_{\beta} e^{-\tilde{y}}\left(\mathcal{I}_{\frac{t+t_{2}}{2}}(\beta \bar{K})\right)^{-1 / \beta}\right)\right]}\right.\right. \\
+\mathbb{E}^{(e)}\left[\left(1-e^{-z C_{\beta} e^{-\tilde{y}}\left(\mathcal{I}_{\delta}(\beta \bar{K})\right)^{-1 / \beta}}\right)\right] \mathbb{P}^{(e)}\left(\frac{t-t_{2}}{2}<\tau_{-\tilde{y}} \leq t-\delta\right) .
\end{aligned}
$$

Thus from (4.6), (4.7) and (1.9), we have

$$
\begin{aligned}
\mathbb{E}^{(e)}[ & \left.\left(1-e^{-z C_{\beta}\left(\mathcal{I}_{t}(\beta(\bar{K}))\right)^{-1 / \beta}}\right), \tau_{-\tilde{y}} \leq t-\delta\right] \\
& \leq 2^{2-\rho} \frac{\Gamma(1+\rho) C_{\beta, q, \rho}\left(z e^{-\tilde{y}}\right)}{\rho}\left(t+t_{2}\right)^{\rho-1} \ell\left(\frac{t+t_{2}}{2}\right) \\
& +\left(A 2^{1-\rho+\delta_{1}}\left(1+\frac{t_{2}-\delta}{2 t_{2}}\right)^{1-\rho+\delta_{1}}-1\right) \hat{V}(\tilde{y})(t-\delta)^{\rho-1} \ell(t-\delta) \\
& \times \mathbb{E}^{(e)}\left[\left(1-e^{-z C_{\beta} e^{-\tilde{y}}\left(\mathcal{I}_{\delta}(\beta \bar{K})\right)^{-1 / \beta}}\right)\right] .
\end{aligned}
$$


Next, we introduce

$$
\begin{aligned}
c_{1}(z, \tilde{y})= & \left(2^{2-\rho} \frac{\Gamma(1+\rho) C_{\beta, q, \rho}\left(z e^{-\tilde{y}}\right)}{\rho}\right. \\
& \left.\vee\left(A 2^{1-\rho+\delta_{1}}\left(1+\frac{t_{2}-\delta}{2 t_{2}}\right)^{1-\rho+\delta_{1}}-1\right) \mathbb{E}^{(e)}\left[1-e^{-z C_{\beta} e^{-\tilde{y}}\left(\mathcal{I}_{\delta}(\beta \bar{K})\right)^{-1 / \beta}}\right]\right) .
\end{aligned}
$$

Therefore from (4.4) and again from Potter's Theorem, we get for $t \geq 3 t_{2}$

$$
\begin{aligned}
\frac{\mathbb{P}_{(z, x)}\left(Z_{t}^{\uparrow}>0, I_{t-\delta}<-y\right)}{t^{\rho-1} \ell(t)} & \leq c_{1}(z, \tilde{y})\left(\frac{\ell\left(\frac{t+t_{2}}{2}\right)}{\ell(t)}+\left(1-\frac{\delta}{3 t_{2}}\right)^{\rho-1} \widehat{V}(y+x) \frac{\ell(t-\delta)}{\ell(t)}\right) \\
& \leq c_{1}(z, \tilde{y}) A\left(2^{\delta_{1}}+\left(1-\frac{\delta}{3 t_{2}}\right)^{\rho-1-\delta_{1}} \widehat{V}(y+x)\right) .
\end{aligned}
$$

Finally, we observe that the map $x \mapsto x^{q}\left(1-e^{-z C_{\beta} e^{-\tilde{y}} x^{-1 / \beta}}\right)$ is bounded and goes to 0 as $y$ goes to $\infty$. Similarly the r.v. $1-e^{-z C_{\beta} e^{-\tilde{y}}\left(\mathcal{I}_{\delta}(\beta \bar{K})\right)^{-1 / \beta}}$ is bounded by one and goes to $0, \mathbb{P}^{(e)}$-a.s., as $y$ goes to $\infty$. Thus by the Dominated Convergence Theorem, we have

$$
C_{\beta, q, \rho}\left(z e^{-\tilde{y}}\right)=\int_{0}^{\infty} x^{q}\left(1-e^{-z C_{\beta} e^{-\tilde{y}} x^{-1 / \beta}}\right) \nu_{q, \rho}(\mathrm{d} x) \underset{y \rightarrow \infty}{\longrightarrow} 0,
$$

and

$$
\mathbb{E}^{(e)}\left[1-e^{-z C_{\beta} e^{-\tilde{y}}\left(\mathcal{I}_{\delta}(\beta \bar{K})\right)^{-1 / \beta}}\right] \underset{y \rightarrow \infty}{\longrightarrow} 0
$$

In other words $c_{1}(z, \tilde{y}) \rightarrow 0$, as $y$ increases. This implies that

$$
\lim _{y \rightarrow \infty} \limsup _{t \rightarrow \infty} \frac{\mathbb{P}_{(z, x)}\left(Z_{t}^{\uparrow}>0, I_{t-\delta}<-y\right)}{t^{\rho-1} \ell(t)}=0
$$

since $\widehat{V}(y)=\mathcal{O}(y)$ and for $y \rightarrow \infty$.

Using Lemmas 4.1 and 4.2, we are now able to conclude the proof of our main result.

Proof of Theorem 1.2. Let $z, x, \varepsilon>0$ and $\delta \in(0,1)$. From Lemma 4.2, we can choose $y$ such that for $t$ large enough,

$$
\mathbb{P}_{(z, x)}\left(Z_{t}>0, I_{t-\delta}<-y\right) \leq \varepsilon \mathbb{P}_{(z, x+y)}\left(Z_{t}>0, I_{t}>0\right)
$$

Hence we deduce

$$
\begin{aligned}
\mathbb{P}_{z}\left(Z_{t}>0\right) & =\mathbb{P}_{(z, x)}\left(Z_{t}>0, I_{t-\delta}>0\right) \\
& +\mathbb{P}_{(z, x)}\left(Z_{t}>0,-y<I_{t-\delta} \leq 0\right)+\mathbb{P}_{(z, x)}\left(Z_{t}>0, I_{t-\delta} \leq-y\right) \\
& \leq \mathbb{P}_{(z, x+y)}\left(Z_{t}>0, I_{t-\delta}>0\right)+\varepsilon \mathbb{P}_{(z, x+y)}\left(Z_{t}>0, I_{t}>0\right) \\
& \leq \mathbb{P}_{(z, x+y)}\left(Z_{t-\delta}>0, I_{t-\delta}>0\right)+\varepsilon \mathbb{P}_{(z, x+y)}\left(Z_{t}>0, I_{t}>0\right) \\
& \leq\left(\frac{\mathbb{P}_{(z, x+y)}\left(Z_{t-\delta}>0, I_{t-\delta}>0\right)}{\mathbb{P}_{(z, x+y)}\left(Z_{t}>0, I_{t}>0\right)}+\varepsilon\right) \mathbb{P}_{(z, x+y)}\left(Z_{t}>0, I_{t}>0\right)
\end{aligned}
$$


From Lemma 4.1, we know

$$
\begin{aligned}
\mathbb{P}_{(z, x+y)}\left(Z_{t}>0, I_{t}>0\right) & \sim c(z, x+y) \mathbb{P}_{x+y}^{(e)}\left(I_{t}>0\right) \\
& \sim c(z, x+y) \widehat{V}(x+y) t^{-(1-\rho)} \ell(t), \quad \text { as } t \rightarrow \infty .
\end{aligned}
$$

From Potter's Theorem (see Thm. 1.5.6 in Bingham et al. [10]), we deduce that for any $A>1$ and $\delta_{1}>0$ there exists $t_{1}:=t_{1}\left(A_{1}, \delta_{1}\right)$ such that

$$
\mathbb{P}_{z}\left(Z_{t}>0\right) \leq\left(A\left(1+\frac{\delta}{t_{1}-\delta}\right)^{1-\rho+\delta_{1}}+\varepsilon\right) \mathbb{P}_{(z, x+y)}\left(Z_{t}>0, I_{t}>0\right)
$$

In other words, for every $\varepsilon>0$, there exists $y^{\prime}>0$ such that

$$
\mathbb{P}_{\left(z, y^{\prime}\right)}\left(Z_{t}>0, I_{t}>0\right) \leq \mathbb{P}_{z}\left(Z_{t}>0\right) \leq\left(A\left(1+\frac{\delta}{t_{1}-\delta}\right)^{1-\rho+\delta_{1}}+\varepsilon\right) \mathbb{P}_{\left(z, y^{\prime}\right)}\left(Z_{t}>0, I_{t}>0\right)
$$

for some $A>1$ and $\delta_{1}>1$. Recall that $y^{\prime}$ is a sequence which may depend on $z$ and $\varepsilon$ and goes to infinity as $\varepsilon$ goes to 0 . Thus, let us take any sequence $y(z, \varepsilon)$ satisfying for any $z, \varepsilon>0$

$$
\begin{aligned}
\mathbb{P}_{(z, y(z, \varepsilon))}\left(Z_{t}>0, I_{t}>0\right) & \leq \mathbb{P}_{z}\left(Z_{t}>0\right) \\
& \leq\left(A\left(1+\frac{\delta}{t_{1}-\delta}\right)^{1-\rho+\delta_{1}}+\varepsilon\right) \mathbb{P}_{(z, y(z, \varepsilon))}\left(Z_{t}>0, I_{t}>0\right),
\end{aligned}
$$

and prove that

$$
C(z):=\lim _{\varepsilon \rightarrow 0} c(z, y(z, \varepsilon)) \widehat{V}(y(z, \varepsilon))
$$

exists and is positive and finite. Dividing equation (4.9) by $t^{\rho-1} \ell(t)$ and using (4.8), we deduce

$$
\begin{aligned}
0<c(z, y(z, \varepsilon)) \widehat{V}(y(z, \varepsilon)) & \leq \liminf _{t \rightarrow \infty} \frac{\mathbb{P}_{z}\left(Z_{t}>0\right)}{t^{\rho-1} \ell(t)} \\
& \leq\left(A\left(1+\frac{\delta}{t_{1}-\delta}\right)^{1-\rho+\delta_{1}}+\varepsilon\right) c(z, y(z, \varepsilon)) \widehat{V}(y(z, \varepsilon))<\infty
\end{aligned}
$$

Now, letting $\delta$ goes to 0 and then $\varepsilon$ tends to 0 , we get

$$
\begin{aligned}
0<\limsup _{\varepsilon \rightarrow 0} c(z, y(z, \varepsilon)) \widehat{V}(y(z, \varepsilon)) & \leq \liminf _{t \rightarrow \infty} \frac{\mathbb{P}_{z}\left(Z_{t}>0\right)}{t^{-(1-\rho) \ell(t)}} \\
& \leq \liminf _{\varepsilon \rightarrow 0}(A+\varepsilon) c(z, y(z, \varepsilon)) \widehat{V}(y(z, \varepsilon)) \\
& =A \liminf _{\varepsilon \rightarrow 0} c(z, y(z, \varepsilon)) \widehat{V}(y(z, \varepsilon))<\infty .
\end{aligned}
$$

Since $A$ can be taken arbitrarily close to 1 , the inferior and superior limits (when $\varepsilon$ goes to 0 ) of the sequence $c(z, y(z, \varepsilon)) \widehat{V}(y(z, \varepsilon))$ are thus equal, positive and finite. We thus deduce that this sequence has a limit $C(z)$ 
when $\varepsilon$ goes to 0 , which is also the limit of

$$
\frac{\mathbb{P}_{z}\left(Z_{t}>0\right)}{t^{-(1-\rho) \ell(t)}} \quad \text { when } t \text { goes to } \infty
$$

and obtain

$$
\mathbb{P}_{z}\left(Z_{t}>0\right) \sim C(z) t^{-(1-\rho)} \ell(t)
$$

This completes the proof.

\section{Appendix A.}

We provide in Appendix the proof of some technical results for the sake of completeness.

Lemma A.1. If (1.1) holds then the process $Z$ is conservative, i.e.

$$
\mathbb{P}_{z}\left(Z_{t}<\infty\right)=1, \quad \text { for any } \quad t \geq 0
$$

and any starting point $z \geq 0$.

Proof. Recall that there exists a functional $v_{t}(s, \lambda, \bar{K})$ which is the unique solution of the backward differential equation (1.13) which determines the law of the reweighted process $\left(Z_{t} e^{-\bar{K}_{t}}, t \geq 0\right)$ as follows,

$$
\mathbb{E}_{(z, x)}\left[\exp \left\{-\lambda Z_{t} e^{-\bar{K}_{t}}\right\}\right]=\mathbb{E}^{(e)}\left[\exp \left\{-z v_{t}\left(0, \lambda e^{-x}, \bar{K}\right)\right\}\right] .
$$

If we let $\lambda$ go to 0 in the previous identity, we deduce

$$
\mathbb{P}_{z}\left(Z_{t}<\infty\right)=\lim _{\lambda \downarrow 0} \mathbb{E}_{(z, x)}\left[\exp \left\{-\lambda Z_{t} e^{-\bar{K}_{t}}\right\}\right]=\mathbb{E}^{(e)}\left[\exp \left\{-z \lim _{\lambda \downarrow 0} v_{t}\left(0, \lambda e^{-x}, \bar{K}\right)\right\}\right],
$$

where the limits are justified by monotonicity and dominated convergence. This implies that the process $Z$ is conservative if and only if

$$
\lim _{\lambda \downarrow 0} v_{t}\left(0, \lambda e^{-x}, \bar{K}\right)=0
$$

for every positive $t$. Let us recall that the function $\Phi(\lambda)$ equals $\lambda^{-1} \psi_{0}(\lambda)$ and observe that $\Phi(0)=\psi_{0}^{\prime}(0+)=0$ (see (3.7)). Since $\psi_{0}$ is convex and non-negative, we deduce that $\Phi$ is increasing. Finally, if we solve equation (1.13) with $\psi_{0}(\lambda)=\lambda \Phi(\lambda)$, we get

$$
v_{t}\left(s, \lambda e^{-x}, \bar{K}\right)=\lambda e^{-x} \exp \left\{-\int_{s}^{t} \Phi\left(e^{-\bar{K}_{r}} v_{t}\left(r, \lambda e^{-x}, \bar{K}\right)\right) \mathrm{d} r\right\} .
$$

Therefore, since $\Phi$ is increasing and $\Phi(0)=0$, we have

$$
0 \leq \lim _{\lambda \rightarrow 0} v_{t}\left(0, \lambda e^{-x}, \bar{K}\right)=\lim _{\lambda \rightarrow 0} \lambda e^{-x} \exp \left\{-\int_{0}^{t} \Phi\left(e^{-\bar{K}_{r}} v_{t}\left(r, \lambda e^{-x}, \bar{K}\right)\right) \mathrm{d} r\right\} \leq \lim _{\lambda \rightarrow 0} \lambda e^{-x}=0,
$$

implying that $Z$ is conservative. 
Proof of Proposition 1.1. By Itô's formula, we have

$$
Z_{t} e^{-\bar{K}_{t}}=Z_{0}+\int_{0}^{t} e^{-\bar{K}_{s}} \sqrt{2 \gamma^{2} Z_{s}} \mathrm{~d} B_{s}^{(b)}+\int_{0}^{t} \int_{(0, \infty)} \int_{0}^{Z_{s-}} z e^{-\bar{K}_{s-}} \widetilde{N}^{(b)}(\mathrm{d} s, \mathrm{~d} z, \mathrm{~d} u),
$$

$\mathbb{P}$-a.s. Then, for $\mathbb{P}^{(e)}$ almost every $w^{(e)}$, we consider

$$
Y_{t}^{w^{(e)}}=Y_{0}^{w^{(e)}}+M_{t}^{w^{(e)}}+N_{t}^{w^{(e)}}+W_{t}^{w^{(e)}} \quad \mathbb{P}^{(b)} \text {-a.s. }
$$

for any $t \geq 0$, where $Y_{t}^{w^{(e)}}=Z_{t}\left(w^{(e)},.\right) \exp \left(-\bar{K}_{t}\left(w^{(e)}\right)\right)$ and

$$
\begin{aligned}
M_{t}^{w^{(e)}} & =\int_{0}^{t} e^{-\bar{K}_{s}\left(w^{(e)}\right)} \sqrt{2 \gamma^{2} Z_{s}} \mathrm{~d} B_{s}^{(b)} \\
N_{t}^{w^{(e)}} & =\int_{0}^{t} \int_{(0,1]} \int_{0}^{Z_{s-}} z e^{-\bar{K}_{s-}\left(w^{(e)}\right)} \widetilde{N}^{(b)}(\mathrm{d} s, \mathrm{~d} z, \mathrm{~d} u), \\
W_{t}^{w^{(e)}} & =\int_{0}^{t} \int_{[1, \infty)} \int_{0}^{Z_{s-}} z e^{-\bar{K}_{s-}\left(w^{(e)}\right)} \widetilde{N}^{(b)}(\mathrm{d} s, \mathrm{~d} z, \mathrm{~d} u),
\end{aligned}
$$

are $\left(\Omega^{(b)}, \mathcal{F}^{(b)}, \mathbb{P}^{(b)}\right)$ local martingales. Let us now check that $Y^{w^{(e)}}$ is a $\left(\Omega^{(b)}, \mathcal{F}^{(b)}, \mathbb{P}^{(b)}\right)$ martingale by proving that the first moment of its supremum on $[0, T]$ is finite, for any $T>0$. We consider the first time $\tau_{N}$ when $Y^{w^{(e)}}$ goes beyond $N$. Using $|x| \leq 1+x^{2}$ and that $Y^{w^{(e)}}$ is bounded before the stopping time $\tau_{N}$, we get

$$
\mathbb{E}\left[\sup _{s<t \wedge \tau_{N}} Y_{s}^{w^{(e)}}\right] \leq 2+\mathbb{E}\left[\sup _{s<t \wedge \tau_{N}}\left(M_{t}^{w^{(e)}}\right)^{2}\right]+\mathbb{E}\left[\sup _{s<t \wedge \tau_{N}}\left(N_{t}^{w^{(e)}}\right)^{2}\right]+\mathbb{E}\left[\sup _{s<t \wedge \tau_{N}}\left|W_{t}^{w^{(e)}}\right|\right] .
$$

Using that $\sup _{[0, T]}\left|\bar{K}\left(w^{(e)}\right)\right|<\infty$, we obtain that $e^{-\bar{K}\left(w^{(e)}\right)}$ is bounded before time $T$ (and the bound does not depend on $N$ ). Thanks to Doob inequality applied to the stopped martingales, there exists $C_{7}$ (which does not depend on $N$ ) such that for any $t \leq T$,

$$
\begin{aligned}
\mathbb{E}\left[\sup _{s<t \wedge \tau_{N}}\left(M_{s}^{w^{(e)}}\right)^{2}\right] & \leq C_{7} \int_{0}^{t} \mathbb{E}\left[\sup _{s<t \wedge \tau_{N}} Y_{s}^{w^{(e)}}\right] \mathrm{d} s, \\
\mathbb{E}\left[\sup _{s<t \wedge \tau_{N}}\left(N_{s}^{w^{(e)}}\right)^{2}\right] & \leq C_{7} \int_{[0,1]} z^{2} \mu(\mathrm{d} z) \int_{0}^{t} \mathbb{E}\left[\sup _{s<t \wedge \tau_{N}} Y_{s}^{w^{(e)}}\right] \mathrm{d} s, \\
\mathbb{E}\left[\sup _{s<t \wedge \tau_{N}}\left|W_{s}^{w^{(e)}}\right|\right] & \leq C_{7} \int_{[1, \infty]} z \mu(\mathrm{d} z) \int_{0}^{t} \mathbb{E}\left[\sup _{s<t \wedge \tau_{N}} Y_{s}^{w^{(e)}}\right] \mathrm{d} s .
\end{aligned}
$$

Then Gronwall's Lemma ensures that there exists $C(T)$ such that for any $t \leq T$ and $N \geq 1$, $\mathbb{E}\left[\sup _{s<t \wedge \tau_{N}} Y_{s}^{w^{(e)}}\right] \leq C(T)$. Letting $N$ go to infinity completes the proof.

Acknowledgements. The authors are very grateful to the anonymous referees for their thorough review. This work was partially funded by the Chair "Modélisation Mathématique et Biodiversité" of VEOLIA-Ecole Polytechnique-MNHN-F.X and ANR ABIM 16-CE40-0001. JCP acknowledge support from the Royal Society and CONACyT (CB-250590). This work was concluded whilst JCP was on sabbatical leave holding a David Parkin Visiting Professorship at the University of Bath. He gratefully acknowledges the kind hospitality of the Department and University. 


\section{REFERENCES}

[1] V.I. Afanasyev, C. Böinghoff, G. Kersting and V.A. Vatutin, Limit theorems for weakly subcritical branching processes in random environment. J. Theor. Probab. 25 (2012) 703-732.

[2] V.I. Afanasyev, J. Geiger, G. Kersting and V.A. Vatutin, Criticality for branching processes in random environment. Ann. Probab. 33 (2005) 645-673.

[3] V. Bansaye and J. Berestycki, Large deviations for branching processes in random environment. Markov Proc. Rel. Fields 15 (2009) 493-524.

[4] V. Bansaye, M.-E. Caballero and S. Méléard, Scaling limits of population and evolution processes in random environment. Electr. J. Probab. 24 (2019).

[5] V. Bansaye, J.C. Pardo and C. Smadi, On the extinction of continuous state branching processes with catastrophes. Electr. J. Probab. 18 (2013) 1-36.

[6] V. Bansaye and F. Simatos, On the scaling limits of Galton-Watson processes in varying environments. Electron. J. Probab. 20 (2015) 1-36.

[7] J. Bertoin, Lévy processes. Vol. 121 of Cambridge Tracts in Mathematics. Cambridge University Press, Cambridge (1996).

[8] J. Bertoin and R. Doney, Spitzer's condition for random walks and Lévy processes. Annales de l'IHP Probabilités et Statistiques 33 (1997) 167-178.

[9] N.H. Bingham, Continuous branching processes and spectral positivity. Stochastic Process. Appl. 4 (1976) $217-242$.

[10] N.H. Bingham, C.M. Goldie and J.L. Teugels, Vol. 27 of Regular variation. Cambridge University Press (1989).

[11] C. Boeinghoff and M. Hutzenthaler, Branching diffusions in random environment. Markov Proc. Rel. Fields 18 (2012) $269-310$.

[12] C. Böinghoff, E. Dyakonova, G. Kersting and V. Vatutin, Branching processes in random environment which extinct at a given moment. Markov Proc. Rel. Fields 16 (2010) 329-350.

[13] L. Chaumont, Conditionings and path decompositions for Lévy processes. Stochastic Process. Appl. 64 (1996) $39-54$.

[14] L. Chaumont and R.A. Doney, On Lévy processes conditioned to stay positive. Electron. J. Probab 10 (2005) $948-961$.

[15] D.A. Dawson and Z. Li, Stochastic equations, flows and measure-valued processes. Ann. Probab. 40 (2012) $813-857$.

[16] R.A. Doney, Fluctuation Theory for Lévy Processes: Ecole D'Eté de Probabilités de Saint-Flour XXXV-2005. Springer (2007).

[17] D.R. Grey, Asymptotic behaviour of continuous time, continuous state-space branching processes. J. Appl. Probab. 11 (1974) 669-677.

[18] A. Grimvall, On the convergence of sequences of branching processes. Ann. Probab. 2 (1974) 1027-1045.

[19] H. He, Z. Li and W. Xu, Continuous-state branching processes in Lévy random environments. J. Theor. Probab. (2018) 1-23.

[20] M. Jirina, Stochastic branching processes with continuous state space. Czechoslovak Math. J. 8 (1958) $292-313$.

[21] T.G. Kurtz, Diffusion approximations for branching processes, in Vol. 5 of Branching processes (Conf., Saint Hippolyte, Que., 1976) (1978) 269-292.

[22] J. Lamperti, Continuous state branching processes. Bull. Amer. Math. Soc. 73 (1967) 382-386.

[23] J. Lamperti, The limit of a sequence of branching processes. Z. Wahrscheinlichkeitstheorie und Verw. Gebiete 7 (1967) $271-288$.

[24] Z. Li and W. Xu, Asymptotic results for exponential functionals of Lévy processes. Stochastic Process. Appl. (2017).

[25] S. Palau and J.C. Pardo, Continuous state branching processes in random environment: the Brownian case. Stochastic Processes Appl. 127 (2017) 957-994.

[26] S. Palau and J.C. Pardo, Branching processes in a Lévy random environment. Acta Appl. Math. 153 (2018) 55-79.

[27] S. Palau, J.C. Pardo and C. Smadi, Asymptotic behaviour of exponential functionals of Lévy processes with applications to random processes in random environment. ALEA Lat. Am. J. Probab. Math. Stat. 13 (2016) 1235-1258.

[28] P. Patie and M. Savov, Bernstein-gamma functions and exponential functionals of Lévy processes. Electron. J. Probab. 23 (2018).

[29] W.L. Smith and W.E. Wilkinson, On branching processes in random environments. Ann. Math. Statist. 40 (1969) 814-827. 